\title{
Use of Interventions to Overcome Medication Non-Adherence
}

\author{
Saibal Kumar Saha, Sikkim Manipal Institute of Technology, Sikkim Manipal University, Gangtok, India \\ (iD) https://orcid.org/0000-0002-7842-698X \\ Anindita Adhikary, Sikkim Manipal Institute of Technology, Sikkim Manipal University, Gangtok, India \\ Ajeya Jha, Sikkim Manipal Institute of Technology, Sikkim Manipal University, Gangtok, India \\ (iD) https://orcid.org/0000-0003-0491-5008
}

Vijay Kumar Mehta, Sikkim Manipal Institute of Medical Sciences, Sikkim Manipal University, Gangtok, India

\begin{abstract}
Medication non-adherence is a global problem and has existed for centuries. Patients have paid a very high price for their behaviour of non-adherence in the form of impaired cost, prolonged diseases, a burden to family, or even by their lives. In the era of science and technology where there is a solution for every odd problem, the issue of medication non-adherence can also find a remedy. This paper tries to highlight the factors of non-adherence and looks for solutions through various forms of technology. The review of different published literature highlights the findings of researchers and tries to assimilate a solution for addressing the prolonged problem of medication non-adherence.
\end{abstract}

\section{KEYWORDS}

Drug Regime, eHealth, Medication Adherence, Medication Compliance, Medication Schedule, mHealth, NonAdherence, Non-Adherence Factors

\section{INTRODUCTION}

Medication non-adherence is a global health care problem which has persisted over the years (De Geest et al., 2019). It is complex and costly, contributing to deprived treatment results and erosion of health care resources (Arbuckle et al., 2019). Proper adherence to medications is very important for assuaging symptoms and curing disease (Yap et al., 2016). According to Kravitz \& Melnikow, (2004) effects of non-adherence on health care costs and adverse events differ due to type of disease, degree of non-adherence and treatment. Approximately 33\% to $69 \%$ of medication-related hospitalizations are due to poor adherence. Annual costings of medication non-adherence range from nearly US $\$ 100$ billion to U\$290 billion in the USA (Osterberg \& Blaschke, 2005), €1.25 billion in Europe and approximately \$A7 billion in Australia (Cutler et al., 2018). Patient's health is adversely affected by medication non-adherence (Ruddy et al., 2009). It can negatively impact a patient's relationship with the physician or health care provider (Waterhouse et al., 1993). To produce desired therapeutic effect, 
enough medication needs to be taken by patients. Hence, intake of $\geq 80 \%$ of prescribed medications is referred to as adequate adherence. Although, according to Vik et al., (2004) this threshold is arbitrary.

The paper is broadly divided into six sections. In the first section, a brief about medication adherence and non-adherence behaviour of patients are addressed. In the second section, different factors associated with non-adherence is reckoned and tabulated. The third section highlights the measurement techniques of non-adherence. In the fourth section, different techniques used for addressing the problem of medication non-adherence is addressed. This section is further divided into six subsections each emphasizing the studies that have been done on a technique for increasing adherence viz: traditional reminders, active reminders, phone call, short message service (SMS), mobile applications and tailored reminders. The fifth section gives a brief about the benefits of medication adherence while the sixth section enumerates the behavioural models used to encompass medication non-adherence behaviour of patients.

\section{MEDICATION ADHERENCE}

According to Cramer et al., (2008) adherence is "the extent to which a patient acts in accordance with the prescribed interval and dose of a dosing regimen." One of the causal factors of preventable adverse events is proper medication adherence. According to Doggrell, (2010) and George et al., (2008) the rate of medication adherence varies from $30 \%$ to $100 \%$ which are influenced by patient characteristics and diversity of socio-medical constructs. Studies showed adherence rates of 30$70 \%$ for patients with asthma, 5-90\% for patients with hypertension and $20-90 \%$ for patients with schizophrenic. Ho et al., (2006) worked on diabetic patients and reported that all-cause hospitalization was $23.2 \%$ for non-adherent patients compared to $19.2 \%$ for adherent. Sokol et al., (2005) classified medication adherence rates into 5 subsets: $0-20 \%, 21-40 \%, 41-60 \%, 61-80 \%$, and $>80 \%$. The study was conducted for congestive heart failure (CHF), diabetes, hypercholesterolemia and hypertension patients below the age group of 65 years. It was found that hospitalization risk and healthcare costs was inversely correlated with each of the levels of medication adherence. The reports highlighted that hospitalization risk for diabetes was $13 \%$ for adherent and $24 \%$ for non-adherent, CHF (57\% vs. 63\%), hypercholesterolemia (12\% vs. 14\%) and hypertension (19\% vs. 23\%). Sullivan, (1990) attributed $2.9 \%$ to $19.5 \%$ of hospital admissions to medication non-adherence. The work of Lau \& $\mathrm{Nau}$, (2004) on type 2 diabetes reveal that non-adherent patients were hospitalized more frequently than adherent $(\geq 80 \%)$ patients. Hospitalization rate for $100 \%$ adherent patients was $4.1 \%$, which increased to $14.8 \%$ when adherence dropped below $40 \%$. Based on Medicare and Medicaid pharmacy claims data, Esposito et al., (2009) performed a cohort study on heart failure patients' and found that adherent patients were 13\% less likely to have hospital admissions and medical costs were $15 \%$ less. Zed et al., (2008) also conducted a study in Canadian tertiary care hospital and found that 3\% of Emergency Department (ED) visits were due to non-adherence.

\section{Non-Adherence}

Non-adherence can be broadly classified into two categories: intentional or unintentional (Nair et al., 2011) and it varies among patients. Unintentional non-adherence patients plan to take their medication as instructed but are unable to do so due to forgetfulness, carelessness or some other reason. Unintentional non-adherence is swayed by characteristics of patients, patient-provider issues and treatment factors (Wroe, 2002). Intentional non-adherence patients make coherent decision of not taking medication as per instruction due to beliefs, feelings or perceptions. These patients weigh the benefits of treatment against any adverse effects of the treatment (Daleboudt et al., 2011) and choose not to follow the medication regime. In order to mitigate the problems of non-adherence different interventions are required (Garfield et al., 2011) (Xie et al., 2019). Clinical complications due to non-adherence may depend on severity and condition of the disease (DiMatteo et al., 2012). For example, if a patient misses' numerous doses of hypertension medications may not add to extra 
health care service use or result in deleterious consequences, but over dose or under dose of insulin in type 1 diabetes, blood thinners (Warfarin) or anti-infectives for AIDS and TB may end up in austere impediments and extra health care costs.

\section{Factors for Non-Adherence}

The work of Vik et al., (2004) attributes $16 \%$ (range $10.6 \%$ to $58 \%$ ) of medication non adherence to forgetfulness, $47 \%-95 \%$ to adverse effects, $15 \%-52 \%$ to belief that medication is not required in the nonexistence of symptoms. Nair et al., (2011) worked on hypertensive patients and cited the reasons for non-adherence as: forgetfulness $(62 \%)$, side effects $(<1 \%)$, too busy $(3 \%)$, medicare copay barrier (5\%) and other (22\%). However, the study of Doggrell, (2010) reported cost (27.6\%) to be the most common cause of non-adherence and forgetfulness (21.3\%) as the second. The study of Col et al., (1990) reports that out of 315 elderly patients admitted to hospital, $11 \%$ was due to medication nonadherence. Out of these $11 \%, 25 \%$ of non-adherence was due to forgetfulness, $15 \%$ was because of confusion, and $35 \%$ was due to side effects. The other factors identified for non-adherence were: "unnecessary meds" (10\%), cost (15\%), inadequate instruction (10\%), "more is better" (10\%) and "dislikes taking meds" (5\%). The study of Malhotra et al., (2001) in a tertiary care hospital in India attributes $7.6 \%$ of the ED visits to non-adherence and less than $1 \%$ of all ED visits to forgetfulness. In another study, cases of non-adherence leading to hospital admissions cited "inadequate instruction" (25.4\%) as the most common reason. In the study conducted by Faught et al., (2009) non-adherence was associated with higher inpatient days and admission rates. The rate of non-adherence patients was high among uninsured low-income patients generally suffering from chronic disease (FernandezLazaro et al., 2019). Following table lists the different factors for medication non-adherence for various diseases.

\section{Measurement of Non-Adherence}

Non-adherence is difficult to measure precisely and most of the interventions used to reduce it has been mostly unsuccessful (Dayer et al., 2013). Some of the common methods used to measure adherence are patient self-reports, biological monitoring, pill counts, electronic monitoring and refill rates. All these methods have been reported to have limitations and are regarded as proxy measures (Claxton et al., 2001) (Cramer et al., 1989). As patient self-reports depend on memory, they may not always be accurate and are prone to recall bias (Weingart et al., 2008). Pill counts are not reliable as patients may not return the bottles or scrap medicines former to the count (Osterberg \& Blaschke, 2005). Electronic monitoring and refill rates cannot confirm whether patients have actually taken the medication. Biological monitoring (for example sampling urine, blood) is either not feasible and intrusive as for accurate measurement by this method, dose and time administered before sampling are required to be verified. Studies also report that the process of cap removal may not reflect dose ingestion. To calculate adherence rates for dose taking and dose timing, electronic monitoring system is regarded as the best method to measure adherence (Paterson et al., 2002) (Spilker \& Cramer, 1991) (Urquhart, 1997). The work of Browne et al., (2018) highlights the use of edible ingestion sensor to remotely monitor detailed dosing history of medicine intake behaviour of patients. Adherence checking should be done regularly to confirm therapeutic effectiveness, redundant dose and regimen changes should be avoided. Costs related to health care should be controlled and any cases of resistance to therapy should be prevented from emerging (Weingart et al., 2008).

\section{ADDRESSING THE PROBLEM OF MEDICATION NON-ADHERENCE}

In order to improve the medication adherence behaviour of patients most methods aim to alter the behaviour of patients (Conn et al., 2016). Some of the common methods include: education of patients, counselling, using reminders, dosage simplification, reinforcement or amalgamation of these methods (Haynes et al., 1996). Interventions for mitigating the problems of medication non-adherence are 
Table 1. Factors for medication adherence, non-adherence

\begin{tabular}{|c|c|c|c|c|c|c|c|}
\hline \multirow{3}{*}{ SI.No } & \multirow{3}{*}{ Factors } & \multirow{3}{*}{ Disease } & \multicolumn{5}{|c|}{ Citation for: } \\
\hline & & & \multicolumn{2}{|c|}{ Adherence } & \multicolumn{2}{|c|}{ Non adherence } & \multirow{2}{*}{$\begin{array}{c}\text { No } \\
\text { association }\end{array}$} \\
\hline & & & $\begin{array}{c}\text { Positive } \\
\text { association }\end{array}$ & $\begin{array}{c}\text { Negative } \\
\text { association }\end{array}$ & $\begin{array}{c}\text { Positive } \\
\text { association }\end{array}$ & $\begin{array}{c}\text { Negative } \\
\text { association }\end{array}$ & \\
\hline \multirow{4}{*}{1} & \multirow{4}{*}{$\begin{array}{l}\text { Belief about } \\
\text { medication }\end{array}$} & Depression & $\begin{array}{l}\text { (Aikens et } \\
\text { al., 2005) }\end{array}$ & & & & \\
\hline & & Asthma & $\begin{array}{l}\text { (Apter et al., } \\
\text { 2003) (Le } \\
\text { et al., 2008) } \\
\text { (Wells et al., } \\
\text { 2008) }\end{array}$ & & & & \\
\hline & & & $\begin{array}{l}\text { (Gatti et al., } \\
\text { 2009) }\end{array}$ & & & & \\
\hline & & Hypertension & & & & & $\begin{array}{l}\text { (Wang et al., } \\
\text { 2002) }\end{array}$ \\
\hline \multirow{4}{*}{2} & \multirow{4}{*}{$\begin{array}{l}\text { Fear about long- } \\
\text { term effects of } \\
\text { medicine }\end{array}$} & Diabetes & & $\begin{array}{l}\text { (Aikens \& } \\
\text { Piette, 2009) }\end{array}$ & & & \\
\hline & & Hypertension & & $\begin{array}{l}\text { (Aikens \& } \\
\text { Piette, 2009) }\end{array}$ & & & \\
\hline & & & & $\begin{array}{l}\text { (Brown et al., } \\
\text { 2005) }\end{array}$ & & & \\
\hline & & $\begin{array}{l}\text { Coronary heart } \\
\text { disease }\end{array}$ & & $\begin{array}{l}\text { (Khanderia et } \\
\text { al., 2008) }\end{array}$ & & & \\
\hline \multirow[b]{2}{*}{3} & \multirow[b]{2}{*}{$\begin{array}{l}\text { Perception of } \\
\text { having side } \\
\text { effect }\end{array}$} & Diabetes & & $\begin{array}{l}\text { (Chao et al, } \\
\text { 2007) }\end{array}$ & $\begin{array}{l}\text { (Mann et } \\
\text { al., 2009) }\end{array}$ & & \\
\hline & & & & $\begin{array}{l}\text { (Mann et al., } \\
\text { 2007) (Kaplan } \\
\text { et al., 2004) } \\
\text { (Vlasnik et } \\
\text { al., 2005) }\end{array}$ & & & \\
\hline \multirow{2}{*}{4} & \multirow{2}{*}{$\begin{array}{l}\text { Beliefs about } \\
\text { necessity of } \\
\text { medication to } \\
\text { maintain health }\end{array}$} & Diabetes & & & & & $\begin{array}{l}\text { (Aikens et al., } \\
\text { 2005) }\end{array}$ \\
\hline & & Hypertension & & & & & $\begin{array}{l}\text { (Aikens et al., } \\
\text { 2005) }\end{array}$ \\
\hline \multirow{3}{*}{5} & \multirow{3}{*}{$\begin{array}{l}\text { Patient } \\
\text { satisfaction } \\
\text { with health care } \\
\text { provider }\end{array}$} & Diabetes & $\begin{array}{l}\text { (Albright et } \\
\text { al., 2001) }\end{array}$ & & & & $\begin{array}{l}\text { (Trevino et } \\
\text { al., 2005) }\end{array}$ \\
\hline & & Hypertension & & & & & $\begin{array}{l}\text { (Trevino et } \\
\text { al., 2005) } \\
\text { (Kim et al., } \\
\text { 2007) }\end{array}$ \\
\hline & & Hyperlipidemia & & & & & $\begin{array}{l}\text { (Sung et al., } \\
1998 \text { ) }\end{array}$ \\
\hline \multirow[b]{2}{*}{6} & \multirow{2}{*}{$\begin{array}{l}\text { Personal stress } \\
\text { level }\end{array}$} & Diabetes & & & & & $\begin{array}{l}\text { (Albright et } \\
\text { al., 2001) }\end{array}$ \\
\hline & & Hypertension & & & & & $\begin{array}{l}\text { (Thorpe et al., } \\
\text { 2009) (Wang } \\
\text { et al., 2002) }\end{array}$ \\
\hline
\end{tabular}


Table 1. Continued

\begin{tabular}{|c|c|c|c|c|c|c|c|}
\hline \multirow{3}{*}{ Sl.No } & \multirow{3}{*}{ Factors } & \multirow{3}{*}{ Disease } & \multicolumn{5}{|c|}{ Citation for: } \\
\hline & & & \multicolumn{2}{|c|}{ Adherence } & \multicolumn{2}{|c|}{ Non adherence } & \multirow{2}{*}{$\begin{array}{c}\text { No } \\
\text { association }\end{array}$} \\
\hline & & & $\begin{array}{c}\text { Positive } \\
\text { association }\end{array}$ & $\begin{array}{c}\text { Negative } \\
\text { association }\end{array}$ & $\begin{array}{c}\text { Positive } \\
\text { association }\end{array}$ & $\begin{array}{c}\text { Negative } \\
\text { association }\end{array}$ & \\
\hline \multirow{6}{*}{7} & \multirow{6}{*}{ Social support } & Diabetes & $\begin{array}{l}\text { (Albright et } \\
\text { al., 2001) }\end{array}$ & & & & $\begin{array}{l}\text { (Gazmararian } \\
\text { et al., 2006) }\end{array}$ \\
\hline & & Asthma & & & & & $\begin{array}{l}\text { (Apter et al., } \\
\text { 2003) }\end{array}$ \\
\hline & & $\begin{array}{l}\text { Heart transplant } \\
\text { or lung } \\
\text { transplant }\end{array}$ & $\begin{array}{l}\text { (Dew et al., } \\
\text { 2008) }\end{array}$ & & & & \\
\hline & & Hypertension & & & & & $\begin{array}{l}\text { (Gazmararian } \\
\text { et al., 2006) } \\
\text { (Kim et al., } \\
\text { 2007) (Li \& } \\
\text { Froelicher, } \\
\text { 2007) (Wang } \\
\text { et al., 2002) } \\
\text { (Thorpe et al., } \\
\text { 2009) }\end{array}$ \\
\hline & & $\begin{array}{l}\text { Coronary heart } \\
\text { disease }\end{array}$ & $\begin{array}{l}\text { (Mondesir et } \\
\text { al., 2018) }\end{array}$ & & & & \\
\hline & & Depression & $\begin{array}{l}\text { (Voils et al.,, } \\
\text { 2005) }\end{array}$ & & & & \\
\hline 8 & $\begin{array}{l}\text { Social support at } \\
\text { home }\end{array}$ & Heart failure & $\begin{array}{l}\text { (J.-R. Wu et } \\
\text { al., 2008) }\end{array}$ & & & & \\
\hline 9 & Communication & Asthma & & & & & $\begin{array}{l}\text { (Apter et al., } \\
\text { 2003) }\end{array}$ \\
\hline 10 & $\begin{array}{l}\text { Poor patient- } \\
\text { clinician } \\
\text { communication }\end{array}$ & Asthma & & & $\begin{array}{l}\text { (Apter et } \\
\text { al., 1998) }\end{array}$ & & \\
\hline 11 & $\begin{array}{l}\text { Better provider } \\
\text { communication }\end{array}$ & & $\begin{array}{l}\text { (Ogedegbe et } \\
\text { al., 2008) }\end{array}$ & & & & \\
\hline 12 & $\begin{array}{l}\text { Pharmacy } \\
\text { interactions }\end{array}$ & Hyperlipidemia & & & & & $\begin{array}{l}\text { (Sung et al., } \\
1998 \text { ) }\end{array}$ \\
\hline \multirow[b]{2}{*}{13} & \multirow[b]{2}{*}{ Knowledge } & Asthma & $\begin{array}{l}\text { (Wells et al., } \\
\text { 2008) }\end{array}$ & & & & $\begin{array}{l}\text { (Apter et al., } \\
\text { 2003) }\end{array}$ \\
\hline & & Hypertension & & & & & $\begin{array}{l}\text { (Thorpe et al., } \\
\text { 2009) (Wang } \\
\text { et al., 2002) }\end{array}$ \\
\hline 14 & $\begin{array}{l}\text { Negative } \\
\text { knowledge }\end{array}$ & Diabetes & & $\begin{array}{l}\text { (Mann et al., } \\
\text { 2009) }\end{array}$ & & & $\begin{array}{l}\text { (Trevino et } \\
\text { al., 2005) }\end{array}$ \\
\hline 15 & $\begin{array}{l}\text { Lower } \\
\text { knowledge }\end{array}$ & Hypertension & & & $\begin{array}{l}\text { (Kim et al., } \\
\text { 2007) }\end{array}$ & & $\begin{array}{l}\text { (Trevino et } \\
\text { al., 2005) } \\
\text { (Hyre et al., } \\
\text { 2007) }\end{array}$ \\
\hline \multirow{2}{*}{16} & \multirow{2}{*}{ Health literacy } & & & & & & $\begin{array}{l}\text { (Gatti et al., } \\
2009 \text { ) }\end{array}$ \\
\hline & & Hypertension & & & & & $\begin{array}{l}\text { (Thorpe et al., } \\
\text { 2009) }\end{array}$ \\
\hline
\end{tabular}


Table 1. Continued

\begin{tabular}{|c|c|c|c|c|c|c|c|}
\hline \multirow{3}{*}{ Sl.No } & \multirow{3}{*}{ Factors } & \multirow{3}{*}{ Disease } & \multicolumn{5}{|c|}{ Citation for: } \\
\hline & & & \multicolumn{2}{|c|}{ Adherence } & \multicolumn{2}{|c|}{ Non adherence } & \multirow{2}{*}{$\begin{array}{c}\text { No } \\
\text { association }\end{array}$} \\
\hline & & & $\begin{array}{c}\text { Positive } \\
\text { association }\end{array}$ & $\begin{array}{c}\text { Negative } \\
\text { association }\end{array}$ & $\begin{array}{c}\text { Positive } \\
\text { association }\end{array}$ & $\begin{array}{c}\text { Negative } \\
\text { association }\end{array}$ & \\
\hline \multirow{9}{*}{17} & \multirow{9}{*}{ Depression } & Asthma & & & $\begin{array}{l}\text { (Smith et } \\
\text { al., 2006) }\end{array}$ & & $\begin{array}{l}\text { (Apter et al., } \\
\text { 2003) (Janson } \\
\text { et al., 2008) }\end{array}$ \\
\hline & & $\begin{array}{l}\text { Overactive } \\
\text { bladder }\end{array}$ & & & & & $\begin{array}{l}\text { (Balkrishnan } \\
\text { et al., 2006) }\end{array}$ \\
\hline & & Diabetes & & $\begin{array}{l}\text { (Chao et } \\
\text { al., 2007) } \\
\text { (Kilbourne } \\
\text { et al., 2005) } \\
\text { (Jeffrey S } \\
\text { Gonzalez et } \\
\text { al., 2007) } \\
\text { (Nau et } \\
\text { al., 2007) } \\
\text { (J Safren } \\
\text { Gonzalez et } \\
\text { al., 2008) }\end{array}$ & $\begin{array}{l}\text { (Lin et al., } \\
\text { 2004) }\end{array}$ & & $\begin{array}{l}\text { (Balkrishnan } \\
\text { et al., 2003) } \\
\text { (Gazmararian } \\
\text { et al., 2006) }\end{array}$ \\
\hline & & & & $\begin{array}{l}\text { (Brown et al., } \\
\text { 2005) (Gatti } \\
\text { et al., 2009) } \\
\text { (Kaplan et } \\
\text { al., 2004) } \\
\text { (Ogedegbe et } \\
\text { al., 2008) }\end{array}$ & & & $\begin{array}{l}\text { (Insel et al., } \\
\text { 2006) }\end{array}$ \\
\hline & & Hypertension & & $\begin{array}{l}\text { (Siegel et al., } \\
\text { 2007) (Wang } \\
\text { et al., 2002) }\end{array}$ & $\begin{array}{l}\text { (Ogedegbe } \\
\text { et al., 2008) }\end{array}$ & & $\begin{array}{l}\text { (Chapman } \\
\text { et al., 2005) } \\
\text { (Gazmararian } \\
\text { et al., 2006) } \\
\text { (Kim et al., } \\
\text { 2007) }\end{array}$ \\
\hline & & $\begin{array}{l}\text { Coronary heart } \\
\text { disease }\end{array}$ & & & $\begin{array}{l}\text { (Gehi et al., } \\
2005 \text { ) }\end{array}$ & & \\
\hline & & $\begin{array}{l}\text { Kidney } \\
\text { transplant } \\
\text { hemodialysis }\end{array}$ & & $\begin{array}{l}\text { (Cukor et al., } \\
\text { 2009) }\end{array}$ & & & \\
\hline & & $\begin{array}{l}\text { Anticoagulation } \\
\text { therapy }\end{array}$ & & & & & $\begin{array}{l}\text { (Platt et al., } \\
2008)\end{array}$ \\
\hline & & Heart failure & & & & & $\begin{array}{l}\text { (J.-R. Wu et } \\
\text { al., 2008) }\end{array}$ \\
\hline 18 & $\begin{array}{l}\text { Poor patient- } \\
\text { clinician } \\
\text { communication }\end{array}$ & Asthma & & $\begin{array}{l}\text { (Apter et al., } \\
\text { 1998) }\end{array}$ & & & \\
\hline \multirow{3}{*}{19} & \multirow{3}{*}{$\begin{array}{l}\text { Perceived health } \\
\text { status }\end{array}$} & $\begin{array}{l}\text { Overactive } \\
\text { bladder }\end{array}$ & $\begin{array}{l}\text { (Balkrishnan } \\
\text { et al., 2006) }\end{array}$ & & & & $\begin{array}{l}\text { (Janson et al., } \\
2008 \text { ) }\end{array}$ \\
\hline & & Diabetes & & & & & $\begin{array}{l}\text { (Balkrishnan } \\
\text { et al., 2003) }\end{array}$ \\
\hline & & $\begin{array}{l}\text { Anticoagulation } \\
\text { therapy }\end{array}$ & & & & & $\begin{array}{l}\text { (Platt et al., } \\
\text { 2008) }\end{array}$ \\
\hline 20 & $\begin{array}{l}\text { Lower perceived } \\
\text { health status }\end{array}$ & & & & $\begin{array}{l}\text { (Wroth \& } \\
\text { Pathman, } \\
\text { 2006) }\end{array}$ & & \\
\hline
\end{tabular}


Table 1. Continued

\begin{tabular}{|c|c|c|c|c|c|c|c|}
\hline \multirow{3}{*}{ SI.No } & \multirow{3}{*}{ Factors } & \multirow{3}{*}{ Disease } & \multicolumn{5}{|c|}{ Citation for: } \\
\hline & & & \multicolumn{2}{|c|}{ Adherence } & \multicolumn{2}{|c|}{ Non adherence } & \multirow{2}{*}{$\begin{array}{c}\text { No } \\
\text { association }\end{array}$} \\
\hline & & & $\begin{array}{c}\text { Positive } \\
\text { association }\end{array}$ & $\begin{array}{c}\text { Negative } \\
\text { association }\end{array}$ & $\begin{array}{c}\text { Positive } \\
\text { association }\end{array}$ & $\begin{array}{c}\text { Negative } \\
\text { association }\end{array}$ & \\
\hline \multirow{5}{*}{21} & \multirow{5}{*}{$\begin{array}{l}\text { More number } \\
\text { of prescribed } \\
\text { medicines }\end{array}$} & $\begin{array}{l}\text { Overactive } \\
\text { bladder }\end{array}$ & & & & & $\begin{array}{l}\text { (Balkrishnan } \\
\text { et al., 2006) }\end{array}$ \\
\hline & & Diabetes & $\begin{array}{l}\text { (Trevino et } \\
\text { al., 2005) }\end{array}$ & & & & \\
\hline & & Hypertension & $\begin{array}{l}\text { (Trevino et } \\
\text { al., 2005) } \\
\text { (Siegel et al., } \\
2007 \text { ) }\end{array}$ & $\begin{array}{l}\text { (Chapman et } \\
\text { al., 2005) }\end{array}$ & & & $\begin{array}{l}\text { (Patel \& } \\
\text { Taylor, 2002) }\end{array}$ \\
\hline & & Heart failure & & & & & $\begin{array}{l}\text { (J.-R. Wu et } \\
\text { al., 2008) }\end{array}$ \\
\hline & & & & $\begin{array}{l}\text { (Stoehr et al., } \\
\text { 2008) }\end{array}$ & $\begin{array}{l}\text { (Phatak \& } \\
\text { Thomas III, } \\
\text { 2006) }\end{array}$ & & $\begin{array}{l}\text { (Williams et } \\
\text { al., 1998) }\end{array}$ \\
\hline \multirow{5}{*}{22} & \multirow{5}{*}{ Smoking } & $\begin{array}{l}\text { Overactive } \\
\text { bladder }\end{array}$ & & & & & $\begin{array}{l}\text { (Balkrishnan } \\
\text { et al., 2006) }\end{array}$ \\
\hline & & Diabetes & & & & & $\begin{array}{l}\text { (Balkrishnan } \\
\text { et al., 2003) }\end{array}$ \\
\hline & & Hypertension & & $\begin{array}{l}\text { (Bautista, } \\
\text { 2008) }\end{array}$ & & & $\begin{array}{l}\text { (Hyre et al., } \\
2007 \text { ) }\end{array}$ \\
\hline & & $\begin{array}{l}\text { Coronary heart } \\
\text { disease }\end{array}$ & & & $\begin{array}{l}\text { (Gehi et al., } \\
2005 \text { ) }\end{array}$ & & \\
\hline & & $\begin{array}{l}\text { Anticoagulation } \\
\text { therapy }\end{array}$ & & & & & $\begin{array}{l}\text { (Platt et al., } \\
\text { 2008) }\end{array}$ \\
\hline \multirow{2}{*}{23} & \multirow{2}{*}{ Physical activity } & $\begin{array}{l}\text { Overactive } \\
\text { bladder }\end{array}$ & & & & & $\begin{array}{l}\text { (Balkrishnan } \\
\text { et al., 2006) }\end{array}$ \\
\hline & & Diabetes & & & & & $\begin{array}{l}\text { (Balkrishnan } \\
\text { et al., 2003) }\end{array}$ \\
\hline \multirow{4}{*}{24} & \multirow{4}{*}{$\begin{array}{l}\text { Alcohol } \\
\text { consumption }\end{array}$} & $\begin{array}{l}\text { Overactive } \\
\text { bladder }\end{array}$ & & & & & $\begin{array}{l}\text { (Balkrishnan } \\
\text { et al., 2006) }\end{array}$ \\
\hline & & Diabetes & & & & & $\begin{array}{l}\text { (Balkrishnan } \\
\text { et al., 2003) }\end{array}$ \\
\hline & & Hypertension & & $\begin{array}{l}\text { (Bautista, } \\
\text { 2008) }\end{array}$ & & & \\
\hline & & $\begin{array}{l}\text { Coronary heart } \\
\text { disease }\end{array}$ & & & $\begin{array}{l}\text { (Gehi et al., } \\
\text { 2005) }\end{array}$ & & \\
\hline 25 & Hospitalization & Diabetes & & & & & $\begin{array}{l}\text { (Balkrishnan } \\
\text { et al., 2003) }\end{array}$ \\
\hline \multirow{3}{*}{26} & \multirow{3}{*}{$\begin{array}{l}\text { Lesser number } \\
\text { of medical visits }\end{array}$} & Hypertension & & $\begin{array}{l}\text { (Bautista, } \\
\text { 2008) }\end{array}$ & & & $\begin{array}{l}\text { (Schectman et } \\
\text { al., 2002) }\end{array}$ \\
\hline & & Diabetes & & & & & $\begin{array}{l}\text { (Schectman et } \\
\text { al., 2002) }\end{array}$ \\
\hline & & Hyperlipidemia & & & & & $\begin{array}{l}\text { (Schectman et } \\
\text { al., 2002) }\end{array}$ \\
\hline 27 & $\begin{array}{l}\text { Increased } \\
\text { number of clinic } \\
\text { visits }\end{array}$ & Hypertension & $\begin{array}{l}\text { (Vawter et } \\
\text { al., 2008) }\end{array}$ & & & & \\
\hline
\end{tabular}


Table 1. Continued

\begin{tabular}{|c|c|c|c|c|c|c|c|}
\hline \multirow{3}{*}{ SI.No } & \multirow{3}{*}{ Factors } & \multirow{3}{*}{ Disease } & \multicolumn{5}{|c|}{ Citation for: } \\
\hline & & & \multicolumn{2}{|c|}{ Adherence } & \multicolumn{2}{|c|}{ Non adherence } & \multirow{2}{*}{$\begin{array}{c}\text { No } \\
\text { association }\end{array}$} \\
\hline & & & $\begin{array}{c}\text { Positive } \\
\text { association }\end{array}$ & $\begin{array}{c}\text { Negative } \\
\text { association }\end{array}$ & $\begin{array}{c}\text { Positive } \\
\text { association }\end{array}$ & $\begin{array}{c}\text { Negative } \\
\text { association }\end{array}$ & \\
\hline \multirow{4}{*}{28} & \multirow{4}{*}{$\begin{array}{l}\text { Frequency of } \\
\text { concurrent } \\
\text { prescriptions }\end{array}$} & & $\begin{array}{l}\text { (Billups et } \\
\text { al., 2000) }\end{array}$ & & & & \\
\hline & & Diabetes & $\begin{array}{l}\text { (Schectman } \\
\text { et al., 2002) }\end{array}$ & & & & \\
\hline & & Hyperlipidemia & $\begin{array}{l}\text { (Schectman } \\
\text { et al., 2002) }\end{array}$ & & & & \\
\hline & & Hypertension & $\begin{array}{l}\text { (Schectman } \\
\text { et al., 2002) }\end{array}$ & & & & \\
\hline \multirow{3}{*}{29} & \multirow{3}{*}{$\begin{array}{l}\text { Number of days } \\
\text { supplied per } \\
\text { prescription }\end{array}$} & Diabetes & $\begin{array}{l}\text { (Schectman } \\
\text { et al., 2002) }\end{array}$ & & & & \\
\hline & & Hyperlipidemia & $\begin{array}{l}\text { (Schectman } \\
\text { et al., 2002) }\end{array}$ & & & & \\
\hline & & Hypertension & $\begin{array}{l}\text { (Schectman } \\
\text { et al., 2002) }\end{array}$ & & & & \\
\hline 30 & $\begin{array}{l}\text { Number } \\
\text { of chronic } \\
\text { conditions }\end{array}$ & & $\begin{array}{l}\text { (Billups et } \\
\text { al., 2000) }\end{array}$ & & & & \\
\hline 31 & $\begin{array}{l}\text { Mental health } \\
\text { index }\end{array}$ & & & & & & $\begin{array}{l}\text { (Billups et al., } \\
\text { 2000) }\end{array}$ \\
\hline 32 & $\begin{array}{l}\text { Mental-health } \\
\text { status }\end{array}$ & hypertension & & & & & $\begin{array}{l}\text { (Thorpe et al., } \\
\text { 2009) }\end{array}$ \\
\hline 33 & $\begin{array}{l}\text { General health } \\
\text { perception }\end{array}$ & & & & & & $\begin{array}{l}\text { (Billups et al., } \\
\text { 2000) }\end{array}$ \\
\hline 34 & $\begin{array}{l}\text { Physical } \\
\text { functioning } \\
\text { index }\end{array}$ & & & & & & $\begin{array}{l}\text { (Billups et al., } \\
\text { 2000) }\end{array}$ \\
\hline \multirow{2}{*}{35} & \multirow{2}{*}{$\begin{array}{l}\text { More confidence } \\
\text { to take } \\
\text { medicines }\end{array}$} & Diabetes & $\begin{array}{l}\text { (Trevino et } \\
\text { al., 2005) }\end{array}$ & & & & \\
\hline & & Hypertension & $\begin{array}{l}\text { (Trevino et } \\
\text { al., 2005) }\end{array}$ & & & & \\
\hline 36 & $\begin{array}{l}\text { Took the disease } \\
\text { seriously }\end{array}$ & Asthma & $\begin{array}{l}\text { (Chambers et } \\
\text { al., 1999) }\end{array}$ & & & & \\
\hline 37 & $\begin{array}{l}\text { More active } \\
\text { in making } \\
\text { decisions with a } \\
\text { physician }\end{array}$ & Asthma & $\begin{array}{l}\text { (Chambers et } \\
\text { al., 1999) }\end{array}$ & & & & \\
\hline 38 & $\begin{array}{l}\text { Motivation to } \\
\text { maintain health }\end{array}$ & Asthma & & & & & $\begin{array}{l}\text { (Chambers et } \\
\text { al., 1999) }\end{array}$ \\
\hline 39 & $\begin{array}{l}\text { Frustration with } \\
\text { adhering to } \\
\text { therapy }\end{array}$ & Asthma & & & & & $\begin{array}{l}\text { (Chambers et } \\
\text { al., 1999) }\end{array}$ \\
\hline 40 & $\begin{array}{l}\text { Perceived } \\
\text { uncontrollable } \\
\text { barriers to } \\
\text { adhering }\end{array}$ & Asthma & & & & & $\begin{array}{l}\text { (Chambers et } \\
\text { al., 1999) }\end{array}$ \\
\hline
\end{tabular}


Table 1. Continued

\begin{tabular}{|c|c|c|c|c|c|c|c|}
\hline \multirow{3}{*}{ Sl.No } & \multirow{3}{*}{ Factors } & \multirow{3}{*}{ Disease } & \multicolumn{5}{|c|}{ Citation for: } \\
\hline & & & \multicolumn{2}{|c|}{ Adherence } & \multicolumn{2}{|c|}{ Non adherence } & \multirow{2}{*}{$\begin{array}{c}\text { No } \\
\text { association }\end{array}$} \\
\hline & & & $\begin{array}{c}\text { Positive } \\
\text { association }\end{array}$ & $\begin{array}{c}\text { Negative } \\
\text { association }\end{array}$ & $\begin{array}{c}\text { Positive } \\
\text { association }\end{array}$ & $\begin{array}{c}\text { Negative } \\
\text { association }\end{array}$ & \\
\hline 41 & $\begin{array}{l}\text { Fewer perceived } \\
\text { barriers }\end{array}$ & & $\begin{array}{l}\text { (G. C. } \\
\text { Williams et } \\
\text { al., 1998) }\end{array}$ & & & & \\
\hline 42 & $\begin{array}{l}\text { Benefits of } \\
\text { medication } \\
\text { adherence }\end{array}$ & Asthma & & & & & $\begin{array}{l}\text { (Chambers et } \\
\text { al., 1999) }\end{array}$ \\
\hline 43 & $\begin{array}{l}\text { Therapies } \\
\text { initiated closer } \\
\text { together in time }\end{array}$ & Hypertension & $\begin{array}{l}\text { (Chapman et } \\
\text { al., 2005) }\end{array}$ & & & & \\
\hline \multirow{4}{*}{44} & \multirow{4}{*}{ Self-efficacy } & $\begin{array}{l}\text { Coronary heart } \\
\text { disease }\end{array}$ & $\begin{array}{l}\text { (Clark \& } \\
\text { Dodge, 1999) }\end{array}$ & & & & \\
\hline & & & $\begin{array}{l}\text { (Gatti et } \\
\text { al., 2009) } \\
\text { (Náfrádi et } \\
\text { al., 2017) }\end{array}$ & & & & \\
\hline & & Diabetes & $\begin{array}{l}\text { (Shiyanbola } \\
\text { et al., 2018) }\end{array}$ & & & & \\
\hline & & Hypertension & & & & $\begin{array}{l}\text { (Ogedegbe } \\
\text { et al., 2008) }\end{array}$ & $\begin{array}{l}\text { (Kim et al., } \\
\text { 2007) }\end{array}$ \\
\hline 45 & $\begin{array}{l}\text { Loci of control } \\
\text { like internal, } \\
\text { powerful others, } \\
\text { and chance }\end{array}$ & $\begin{array}{l}\text { Kidney } \\
\text { transplant } \\
\text { hemodialysis }\end{array}$ & & & & & $\begin{array}{l}\text { (Cukor et al., } \\
\text { 2009) }\end{array}$ \\
\hline 46 & $\begin{array}{l}\text { Avoidance of } \\
\text { disease triggers }\end{array}$ & Asthma & $\begin{array}{l}\text { (De Smet et } \\
\text { al., 2006) }\end{array}$ & & & & \\
\hline 47 & $\begin{array}{l}\text { Years since } \\
\text { diagnosis }\end{array}$ & Asthma & $\begin{array}{l}\text { (De Smet et } \\
\text { al., 2006) }\end{array}$ & & & & \\
\hline 48 & $\begin{array}{l}\text { Perceived } \\
\text { benefits of } \\
\text { medication }\end{array}$ & Asthma & $\begin{array}{l}\text { (De Smet et } \\
\text { al., 2006) }\end{array}$ & & & & \\
\hline 49 & $\begin{array}{l}\text { More persons } \\
\text { providing } \\
\text { instructions }\end{array}$ & Asthma & $\begin{array}{l}\text { (De Smet et } \\
\text { al., 2006) }\end{array}$ & & & & \\
\hline 50 & $\begin{array}{l}\text { Higher self-rated } \\
\text { severity of } \\
\text { illness }\end{array}$ & Depression & & & $\begin{array}{l}\text { (Sirey et al., } \\
\text { 2001) }\end{array}$ & & \\
\hline 51 & $\begin{array}{l}\text { Self-reported } \\
\text { health status }\end{array}$ & Glaucoma & $\begin{array}{l}\text { (Friedman et } \\
\text { al., 2009) }\end{array}$ & & & & \\
\hline 52 & $\begin{array}{l}\text { Perceived health } \\
\text { outcome }\end{array}$ & Glaucoma & $\begin{array}{l}\text { (Friedman et } \\
\text { al., 2009) }\end{array}$ & & & & \\
\hline
\end{tabular}


Table 1. Continued

\begin{tabular}{|c|c|c|c|c|c|c|c|}
\hline \multirow{3}{*}{ SI.No } & \multirow{3}{*}{ Factors } & \multirow{3}{*}{ Disease } & \multicolumn{5}{|c|}{ Citation for: } \\
\hline & & & \multicolumn{2}{|c|}{ Adherence } & \multicolumn{2}{|c|}{ Non adherence } & \multirow{2}{*}{$\begin{array}{c}\text { No } \\
\text { association }\end{array}$} \\
\hline & & & $\begin{array}{c}\text { Positive } \\
\text { association }\end{array}$ & $\begin{array}{l}\text { Negative } \\
\text { association }\end{array}$ & $\begin{array}{c}\text { Positive } \\
\text { association }\end{array}$ & $\begin{array}{c}\text { Negative } \\
\text { association }\end{array}$ & \\
\hline \multirow{5}{*}{53} & \multirow{5}{*}{$\begin{array}{l}\text { Drug regime } \\
\text { complexity }\end{array}$} & $\begin{array}{l}\text { Coronary heart } \\
\text { disease }\end{array}$ & & $\begin{array}{l}\text { (Gazmararian } \\
\text { et al., 2006) }\end{array}$ & & & \\
\hline & & Diabetes & & $\begin{array}{l}\text { (Gazmararian } \\
\text { et al., 2006) } \\
\text { (Capoccia et } \\
\text { al., 2016) }\end{array}$ & & & \\
\hline & & Hypertension & & $\begin{array}{l}\text { (Gazmararian } \\
\text { et al., 2006) }\end{array}$ & & & \\
\hline & & $\begin{array}{l}\text { Parkinson's } \\
\text { disease }\end{array}$ & & $\begin{array}{l}\text { (Kulkarni et } \\
\text { al., 2008) }\end{array}$ & & & \\
\hline & & & & $\begin{array}{l}\text { (Vlasnik et } \\
\text { al., 2005) }\end{array}$ & & & \\
\hline 54 & $\begin{array}{l}\text { Patients being } \\
\text { comfortable } \\
\text { asking questions } \\
\text { to doctor }\end{array}$ & Hypertension & $\begin{array}{l}\text { (Hyre et al., } \\
2007 \text { ) }\end{array}$ & & & & \\
\hline 55 & $\begin{array}{l}\text { Spending more } \\
\text { time with doctor }\end{array}$ & Hypertension & & $\begin{array}{l}\text { (Hyre et al., } \\
2007 \text { ) }\end{array}$ & & & \\
\hline 56 & $\begin{array}{l}\text { Memory } \\
\text { composite score }\end{array}$ & & & & & & $\begin{array}{l}\text { (Insel et al., } \\
2006 \text { ) }\end{array}$ \\
\hline \multirow{3}{*}{57} & \multirow{3}{*}{$\begin{array}{l}\text { Perceived } \\
\text { disease severity }\end{array}$} & Asthma & $\begin{array}{l}\text { (De Smet et } \\
\text { al., 2006) }\end{array}$ & & $\begin{array}{l}\text { (Janson et } \\
\text { al., 2008) }\end{array}$ & & \\
\hline & & Hypertension & $\begin{array}{l}\text { (Li et al., } \\
2006)\end{array}$ & & & & $\begin{array}{l}\text { (Li \& } \\
\text { Froelicher, } \\
\text { 2007) }\end{array}$ \\
\hline & & & & & $\begin{array}{l}\text { (Mann et } \\
\text { al., 2007) }\end{array}$ & & \\
\hline 58 & $\begin{array}{l}\text { Overuse of } \\
\text { medicines }\end{array}$ & $\begin{array}{l}\text { Coronary heart } \\
\text { disease }\end{array}$ & & $\begin{array}{l}\text { (Khanderia et } \\
\text { al., 2008) }\end{array}$ & & & \\
\hline 59 & $\begin{array}{l}\text { Belief about } \\
\text { disease }\end{array}$ & Hypertension & & & & & $\begin{array}{l}\text { (Kim et al., } \\
\text { 2007) }\end{array}$ \\
\hline 60 & $\begin{array}{l}\text { Perceived } \\
\text { outcome }\end{array}$ & Hypertension & & & & & $\begin{array}{l}\text { (Li \& } \\
\text { Froelicher, } \\
\text { 2007) }\end{array}$ \\
\hline 61 & $\begin{array}{l}\text { Negative } \\
\text { perceived } \\
\text { outcome }\end{array}$ & Diabetes & & & $\begin{array}{l}\text { (Mann et } \\
\text { al., 2009) }\end{array}$ & & \\
\hline 62 & $\begin{array}{l}\text { Expected } \\
\text { treatment } \\
\text { duration }\end{array}$ & & & & $\begin{array}{l}\text { (Mann et } \\
\text { al., 2007) }\end{array}$ & & \\
\hline 63 & $\begin{array}{l}\text { Medicine } \\
\text { characteristics }\end{array}$ & Diabetes & & & $\begin{array}{l}\text { (Mann et } \\
\text { al., 2009) }\end{array}$ & & \\
\hline 64 & Living alone & $\begin{array}{l}\text { Coronary heart } \\
\text { disease }\end{array}$ & & & $\begin{array}{l}\text { (Gehi et al., } \\
2005 \text { ) }\end{array}$ & & \\
\hline
\end{tabular}


Table 1. Continued

\begin{tabular}{|c|c|c|c|c|c|c|c|}
\hline \multirow{3}{*}{ SI.No } & \multirow{3}{*}{ Factors } & \multirow{3}{*}{ Disease } & \multicolumn{5}{|c|}{ Citation for: } \\
\hline & & & \multicolumn{2}{|c|}{ Adherence } & \multicolumn{2}{|c|}{ Non adherence } & \multirow{2}{*}{$\begin{array}{c}\text { No } \\
\text { association }\end{array}$} \\
\hline & & & $\begin{array}{c}\text { Positive } \\
\text { association }\end{array}$ & $\begin{array}{c}\text { Negative } \\
\text { association }\end{array}$ & $\begin{array}{c}\text { Positive } \\
\text { association }\end{array}$ & $\begin{array}{c}\text { Negative } \\
\text { association }\end{array}$ & \\
\hline 65 & $\begin{array}{l}\text { Symptom } \\
\text { severity }\end{array}$ & Diabetes & & $\begin{array}{l}\text { (Gonzalez } \\
\text { et al., 2007) } \\
\text { (Gonzalez et } \\
\text { al., 2008) }\end{array}$ & & & \\
\hline 66 & $\begin{array}{l}\text { Lower beliefs } \\
\text { in drug } \\
\text { effectiveness }\end{array}$ & Osteoporosis & & & $\begin{array}{l}\text { (McHorney } \\
\text { et al.,2007) }\end{array}$ & & \\
\hline 67 & $\begin{array}{l}\text { Lower drug- } \\
\text { safety beliefs }\end{array}$ & Osteoporosis & & & $\begin{array}{l}\text { (McHorney } \\
\text { et al., 2007) }\end{array}$ & & \\
\hline \multirow[b]{2}{*}{68} & \multirow{2}{*}{$\begin{array}{l}\text { Beliefs about } \\
\text { medicines }\end{array}$} & Hypertension & & & $\begin{array}{l}\text { (Mochari et } \\
\text { al., 2007) }\end{array}$ & & \\
\hline & & & & & $\begin{array}{l}\text { (Phatak \& } \\
\text { Thomas III, } \\
\text { 2006) }\end{array}$ & & \\
\hline 69 & $\begin{array}{l}\text { Patient- } \\
\text { physician trust }\end{array}$ & $\begin{array}{l}\text { Inflammatory } \\
\text { bowel disease }\end{array}$ & $\begin{array}{l}\text { (Nguyen et } \\
\text { al., 2009) }\end{array}$ & & & & \\
\hline 70 & $\begin{array}{l}\text { Lower patient- } \\
\text { provider trust }\end{array}$ & & & & $\begin{array}{l}\text { (Wroth \& } \\
\text { Pathman, } \\
\text { 2006) }\end{array}$ & & \\
\hline 71 & $\begin{array}{l}\text { Patient provider } \\
\text { relationship }\end{array}$ & Heart failure & & & & & $\begin{array}{l}\text { (J.-R. Wu et } \\
\text { al., 2008) }\end{array}$ \\
\hline 72 & $\begin{array}{l}\text { Perceived } \\
\text { control over } \\
\text { disease }\end{array}$ & Hypertension & & $\begin{array}{l}\text { (Patel \& } \\
\text { Taylor, 2002) }\end{array}$ & & & $\begin{array}{l}\text { (Thorpe et al., } \\
\text { 2009) }\end{array}$ \\
\hline \multirow{2}{*}{73} & \multirow{2}{*}{ Higher dose } & Hyperlipidemia & & $\begin{array}{l}\text { (Pedan et al., } \\
\text { 2007) }\end{array}$ & & & \\
\hline & & & & & & & $\begin{array}{l}\text { (Stoehr et al., } \\
\text { 2008) }\end{array}$ \\
\hline 74 & $\begin{array}{l}\text { Higher volume } \\
\text { of patients per } \\
\text { physician }\end{array}$ & Hyperlipidemia & & $\begin{array}{l}\text { (Pedan et al., } \\
\text { 2007) }\end{array}$ & & & \\
\hline 75 & $\begin{array}{l}\text { Higher co- } \\
\text { payments }\end{array}$ & Hyperlipidemia & & $\begin{array}{l}\text { (Pedan et al., } \\
\text { 2007) }\end{array}$ & & & \\
\hline 76 & $\begin{array}{l}\text { Number of } \\
\text { prescribed refills }\end{array}$ & Hyperlipidemia & $\begin{array}{l}\text { (Pedan et al., } \\
2007 \text { ) }\end{array}$ & & & & \\
\hline 77 & $\begin{array}{l}\text { Lower mental } \\
\text { function }\end{array}$ & $\begin{array}{l}\text { Anticoagulation } \\
\text { therapy }\end{array}$ & & & $\begin{array}{l}\text { (Platt et al., } \\
\text { 2008) }\end{array}$ & & \\
\hline 78 & $\begin{array}{l}\text { Lower cognitive } \\
\text { function }\end{array}$ & $\begin{array}{l}\text { Anticoagulation } \\
\text { therapy }\end{array}$ & & $\begin{array}{l}\text { (Turner et al., } \\
\text { 2012) }\end{array}$ & $\begin{array}{l}\text { (Platt et al., } \\
\text { 2008) }\end{array}$ & & \\
\hline \multirow{3}{*}{79} & \multirow{3}{*}{$\begin{array}{l}\text { Travel distance } \\
\text { to a pharmacy }\end{array}$} & Diabetes & & & & & $\begin{array}{l}\text { (Schectman et } \\
\text { al., 2002) }\end{array}$ \\
\hline & & Hyperlipidemia & & & & & $\begin{array}{l}\text { (Schectman et } \\
\text { al., 2002) }\end{array}$ \\
\hline & & Hypertension & & & & & $\begin{array}{l}\text { (Schectman et } \\
\text { al., 2002) }\end{array}$ \\
\hline
\end{tabular}


Table 1. Continued

\begin{tabular}{|c|c|c|c|c|c|c|c|}
\hline \multirow{3}{*}{ SI.No } & \multirow{3}{*}{ Factors } & \multirow{3}{*}{ Disease } & \multicolumn{5}{|c|}{ Citation for: } \\
\hline & & & \multicolumn{2}{|c|}{ Adherence } & \multicolumn{2}{|c|}{ Non adherence } & \multirow{2}{*}{$\begin{array}{c}\text { No } \\
\text { association }\end{array}$} \\
\hline & & & $\begin{array}{c}\text { Positive } \\
\text { association }\end{array}$ & $\begin{array}{c}\text { Negative } \\
\text { association }\end{array}$ & $\begin{array}{c}\text { Positive } \\
\text { association }\end{array}$ & $\begin{array}{c}\text { Negative } \\
\text { association }\end{array}$ & \\
\hline \multirow{3}{*}{80} & \multirow{3}{*}{$\begin{array}{l}\text { Once-daily } \\
\text { dosing }\end{array}$} & Diabetes & & & & & $\begin{array}{l}\text { (Schectman et } \\
\text { al., 2002) }\end{array}$ \\
\hline & & Hyperlipidemia & & & & & $\begin{array}{l}\text { (Schectman et } \\
\text { al., 2002) }\end{array}$ \\
\hline & & Hypertension & & & & & $\begin{array}{l}\text { (Schectman et } \\
\text { al., 2002) }\end{array}$ \\
\hline 81 & $\begin{array}{l}\text { Higher } \\
\text { perceived stigma }\end{array}$ & Depression & & & $\begin{array}{l}\text { (Sirey et al., } \\
2001 \text { ) }\end{array}$ & & \\
\hline 82 & $\begin{array}{l}\text { Composite score } \\
\text { for perceived } \\
\text { barriers to } \\
\text { medication }\end{array}$ & Hypertension & & $\begin{array}{l}\text { (Thorpe et al., } \\
\text { 2009) }\end{array}$ & & & \\
\hline 83 & $\begin{array}{l}\text { Emotional well- } \\
\text { being }\end{array}$ & Hypertension & $\begin{array}{l}\text { (Trivedi et } \\
\text { al., 2008) }\end{array}$ & & & & \\
\hline \multirow{2}{*}{84} & \multirow{2}{*}{$\begin{array}{l}\text { Internal locus of } \\
\text { control }\end{array}$} & Depression & & $\begin{array}{l}\text { (Voils et al., } \\
\text { 2005) }\end{array}$ & & & \\
\hline & & & $\begin{array}{l}\text { (Náfrádi et } \\
\text { al., 2017) }\end{array}$ & & & & \\
\hline \multirow{2}{*}{85} & \multirow{2}{*}{$\begin{array}{l}\text { External locus } \\
\text { of control }\end{array}$} & Hypertension & & & & & $\begin{array}{l}\text { (Wang et al., } \\
\text { 2002) }\end{array}$ \\
\hline & & & & $\begin{array}{l}\text { (Náfrádi et al., } \\
\text { 2017) }\end{array}$ & & & \\
\hline 86 & Locus of control & & & & & & $\begin{array}{l}\text { (Williams et } \\
\text { al., 1998) }\end{array}$ \\
\hline 87 & $\begin{array}{l}\text { Difficulty } \\
\text { in affording } \\
\text { medicines }\end{array}$ & Asthma & & $\begin{array}{l}\text { (Wells et al., } \\
\text { 2008) }\end{array}$ & & & \\
\hline 88 & $\begin{array}{l}\text { Inability to get } \\
\text { an appointment }\end{array}$ & Asthma & & $\begin{array}{l}\text { (Wells et al., } \\
\text { 2008) }\end{array}$ & & & \\
\hline 89 & $\begin{array}{l}\text { More perceived } \\
\text { physician } \\
\text { support }\end{array}$ & & $\begin{array}{l}\text { (G. C. } \\
\text { Williams et } \\
\text { al., 1998) }\end{array}$ & & & & \\
\hline 90 & $\begin{array}{l}\text { More patient } \\
\text { autonomy for } \\
\text { improving } \\
\text { health }\end{array}$ & & $\begin{array}{l}\text { (G. C. } \\
\text { Williams et } \\
\text { al., 1998) }\end{array}$ & & & & \\
\hline \multirow{2}{*}{91} & \multirow{2}{*}{$\begin{array}{l}\text { Medicine } \\
\text { frequency }\end{array}$} & Heart failure & & & & & $\begin{array}{l}\text { (J.-R. Wu et } \\
\text { al., 2008) }\end{array}$ \\
\hline & & Kidney failure & & $\begin{array}{l}\text { (Oh et al., } \\
2019 \text { ) }\end{array}$ & & & \\
\hline 92 & Cost & & & $\begin{array}{l}\text { (Vlasnik et } \\
\text { al., 2005) } \\
\text { (Polonsky \& } \\
\text { Henry, 2016) } \\
\text { (Holt et al., } \\
\text { 2013) }\end{array}$ & & & \\
\hline
\end{tabular}




\begin{tabular}{|c|c|c|c|c|c|c|c|}
\hline \multirow{3}{*}{ SI.No } & \multirow{3}{*}{ Factors } & \multirow{3}{*}{ Disease } & \multicolumn{5}{|c|}{ Citation for: } \\
\hline & & & \multicolumn{2}{|c|}{ Adherence } & \multicolumn{2}{|c|}{ Non adherence } & \multirow{2}{*}{$\begin{array}{c}\text { No } \\
\text { association }\end{array}$} \\
\hline & & & $\begin{array}{c}\text { Positive } \\
\text { association }\end{array}$ & $\begin{array}{c}\text { Negative } \\
\text { association }\end{array}$ & $\begin{array}{c}\text { Positive } \\
\text { association }\end{array}$ & $\begin{array}{c}\text { Negative } \\
\text { association }\end{array}$ & \\
\hline 93 & Substance abuse & & & $\begin{array}{l}\text { (Vlasnik et } \\
\text { al., 2005) }\end{array}$ & & & \\
\hline 94 & Pill burden & & & $\begin{array}{l}\text { (Vlasnik et } \\
\text { al., 2005) }\end{array}$ & & & \\
\hline 95 & Quality of life & Heart failure & $\begin{array}{l}\text { (Silavanich et } \\
\text { al., 2019) }\end{array}$ & & & & \\
\hline
\end{tabular}

categorized based on education, behaviour and organization. Attempts to improve the adherence rates is done by lifting barriers allied with medication complication, giving more information, altering patient's environment or incentives and improving communication with care providers (Graves et al., 2009). Vrijens, (2019) proposed a Six Sigma framework consisting of "DMAIC (Define, Measure, Analyse, Improve and Control)" approach to reduce medication non-adherence. Stirratt et al., (2018) identified three stages of adherence: initiation, implementation and persistence and investigated the barriers, facilitators and determinants of each stage. Interventions should be designed considering the specific stage and strategies to target them should be designed accordingly. Following are some of the interventions used for enhancing adherence rates.

\section{Traditional Reminders}

Pill reminder systems include unit-of-use packaging, packaged calendars or weekly pill boxes. For unintentional non-adherence these aids prove to be helpful (Harbig et al., 2012) (Rolnick et al., 2011). In the traditional reminder systems, patients of self-medication are not given access to their adherence data. Any sort of educational information is also not provided to them. The tested method of pill reminder systems has proved to be useful across different medications. However, these systems are cumbersome for complex schedules. These systems only act as passive reminders for medication intake (Mahtani et al., 2011) (Zedler et al., 2011).

\section{Active Reminders}

With the advancement of technology, electronic reminder system (pager, telephone, and audio-visual devices) are being used to proactively deliver reminders. Behaviour of patients can be influenced by mobile phones due to its ease of use, worldwide acceptance and instant information transmitting capabilities (Maddison et al., 2018). These systems if united with alternate behavioural strategies could solve the problems of non-adherence largely. The study of Frick et al., (2001) in Mombasa, Kenya, reveals that use of alarm devices expressively improved non-ART medication adherence rates. According to Fenerty et al., (2012) electronic reminder systems may not be realistic for widespread use.

\section{Phone Call}

By integrating cellular phone and internet based education system, an attempt was made to check the association of blood glucose levels of diabetic patients with the frequency of accessing eMOD (electronic Management of Diabetes) system. The results revealed that the relationship was positive (Noh et al., 2010). Statistics reveal that in developed countries the strategy used for improving adherence for ART in chronic disease by mobile phone is promising (Lester \& Karanja, 2008) (Chang et al., 2008) (Fjeldsoe et al., 2009). For adolescents and young adults interventions by cell phone calls can improve adherence (Sayegh et al., 2018). 


\section{Short Message Service (SMS)}

Teenager patients suffering from asthma gave high ratings for the parameters: usefulness, acceptability and ease of use when they were sent personalized reminders in the form of text messages (Britto et al., 2012). In a study, it was seen that weekly reminders showed significant improvement in adherence, but daily reminders did not. It was presumed that daily messages could have been deliberated as invasive (Pop-Eleches et al., 2011). The study of Thakkar et al., (2016) reports increase in adherence for middle-aged patients with chronic disease. The work of Adler et al., (2017) reveals that use of SMS as intervention to improve medication adherence is useful for patients suffering from cardiovascular disease. According to Oyugi et al., (2007) weekly reminders proved effective in reducing the occurrence of treatment interruptions. While sending messages as reminders one should keep in mind that patients may be using shared mobile phones and their phone or number may change (Shet et al., 2010). However as per Simoni et al., (2006) there is no consensus as to which is the finest approach to improve adherence.

\section{Mobile Applications}

Along with improving the medication adherence rate, smartphone apps can reduce costs of traditional adherence interventions. By using traditional means of telecommunication (SMS) behaviour of patients can be changed and adherence levels can be measured for short term studies (Vervloet et al., 2012). Sending photographs of medication capsules in the phone of patients before ingestion help to acquire precise time measures of adherence (Galloway et al., 2011). Although studies on use of smartphones for improving adherence has been done in clinical settings but studies on empirically testing them are missing (Wu et al., 2011). According to Henny et al., (2018) there is lack of theoretical premise including behavioural theories behind the use of eHealth interventions.

The study conducted by Dayer et al., (2013) reports that the apps present in the market lacks interconnection with other information providing systems. The study lacks to find an app, which is totally assimilated with patient record systems (medical records and community pharmacy prescription databases). An interfacing adherence app with interconnection with pharmacy prescription records could be used by pharmacists to send drug regime reminders to patients in their apps. These interconnected apps may help to solve adherence problems in challenging cases by collective collaboration efforts of patients and pharmacists (Capoccia et al., 2016).

The possibility of connecting medical records with smartphone apps and with other devices can enhance adherence competencies to a great extent (Ahmed et al., 2018). "Proteus Digital Health Feedback System" is an advanced system where solid oral dosage of patient is united with ingestible sensors. The sensors record physiologic parameters post eating and send information to a patch or dermal sensor, which can transmit data to a smartphone. With the help of this system, authentic ingestion of doses can be precisely recorded. An app integrated with such a system can send tailored reminders to patients whenever they miss a dose. Numerous apps and technologies for diabetes self and programmed monitoring have been developed. Studies have reported improvement in diabetes (Cafazzo et al., 2012) (O'grady et al., 2012) and blood pressure control (Morawski et al., 2018) medication adherence. As reminders predominantly focus on unintentional non-adherence, adherence apps require infusion of a well-developed scale for assessing unintentional non-adherence with multimodal strategy to result in unremitting enhancements in adherence (Gadkari \& McHorney, 2012) (Santo et al., 2016).

\section{Tailored Reminders}

The present generation smartphones can perform multi discipline functions and can be used effectively to mitigate the problems of medication non-adherence (Mrosek et al., 2015) (Badawy et al., 2019). These smartphones allow constant access to communication and allow multiple functions to address non-adherence. These apps are available at very low to no cost. Some of the features offered by these 
apps include education of patients, timely reminder for medication and refill, offers a storehouse for patient and medication specific information. The data logs can be used to generate reports, which can be used by patients, caregivers or physicians. With smart engines inbuilt in the apps, disease specific information can be consolidated to provide refined well-targeted behaviour changing messages and information to the patients. Apps can handle complex medication regimens for patients, family members or care givers and help to increase the adherence rates. Some of the apps are also integrated with pharmacies to provide drug related information, pharmacy care contacts or discount coupons (Dayer et al., 2013).

Efforts are being made to integrate health-monitoring devices with smartphones. Several researchers reported that empirical analyses of smartphone apps usage for addressing medication non-adherence is lacking (Wohlers et al., 2009) (Gamble, 2009) (Sposaro \& Tyson, 2009) (Lee et al., 2011) (Delpier et al., 2013) (Kharrazi et al., 2012). The work of Vervloet et al., (2012) reveals an integrated system of electronic pill boxes that monitor box opening events and transmit signals to server. Whenever a patient miss opening a box, SMS text message is sent as a reminder. This system has been reported to improve adherence. "Trans theoretical model stages of change" or "motivational interviewing" models of behavioural change could be used to improve customized messages for improving unintentional and intentional non-adherence (Ogedegbe et al., 2008) (Ficke \& Farris, 2005). Several studies have been done using tailored interventions with smartphones to improve dietary intake of vegetables and fruits (Kerr et al., 2012).

\section{BENEFITS OF ADHERENCE}

Based on data of employer-sponsored insurance beneficiaries, Roebuck et al., (2011) conducted a retrospective review of pharmacy and medical claim records. The study compared the effects of adherence rates for patients with dyslipidemia, diabetes, hypertension and heart failure. It was seen that adherent patients had reduced average annual medical expenditures and lesser inpatient hospital days. In the study of Hepke et al., (2004) cost of ED visits and inpatient hospital admissions for diabetic patients decreased with increase in medication adherence rates.

For prevention and treatment of Human Immunodeficiency Virus (HIV), medication adherence is important for Pre-Exposure Prophylaxis (PrEP) and Antiretroviral Therapy (ART). Baker, (2018) studied the relationship between the different dynamics of social support and physical environment which can affect PrEP and ART adherence across populations. The study also focused on physical settings like home security and privacy. The study reports that PrEP can diminish the risk of HIV, but the medication works well when it is taken regularly (Marrazzo et al., 2015) (Anderson et al., 2012) (Corneli et al., 2014). Association of alcohol and drug use with HIV medication adherence, have been studied extensively over the years. The factors like physical environment settings, social support, partnership types and dynamics have not been studied extensively (Arnsten et al., 2002) (Cook et al., 2001) (Samet et al., 2004) (Hinkin et al., 2004) (Azar et al., 2010) (Chander et al., 2006). According to DiMatteo, (2004) and Protopopescu et al., (2009) partners may offer social support which could encourage positive medication adherence behaviours for improving PrEP or ART adherence.

Based on cause of non-adherence, interventions can be designed to overcome the relevant barrier. Mobile Device Management System (MDMs) are used to address the barriers of mild cognitive deficits forgetfulness, physical impairment (e.g., dexterity, impaired vision), low health literacy and confusion. Based on the complexity of medical regime (dosing frequency and number of prescribed medications) (Hajjar et al., 2007) and factors like food-dosing restrictions (George et al., 2008), MDMs can be used to address non-adherence. MDMs are unlikely to remove the barriers of health beliefs, number of dispensing pharmacies used, negative side effects and cost are not addressed by MDMs. 


\section{BEHAVIOURAL MODELS OF ADHERENCE}

Based on several social cognition models, several models for medication adherence have been made which include health theory of planned behaviour (Ajzen, 1991), social cognitive theory (Bandura, 1986) and belief model (Rosenstock et al., 1988). These models are developed based on the belief of individuals, information interpretation along with their experiences. All these ultimately influence their behaviour (Petrie \& Weinman, 1997). According to Lehane \& McCarthy, (2007) health behaviour of patients is based on rational decisions developed from available information. The dosing behaviour is affected by individual beliefs, culture perspectives, anticipated outcomes, social experiences and different worldviews (Amico et al., 2018). It is a big challenge to improve medication adherence among patients with chronic diseases (Banuelos Mota et al., 2019). Hence, multifaceted interventions are required to improve adherence for long-term treatment (Holloway \& Van Dijk, 2011). Conn \& Ruppar, (2017) suggests that behavioural strategies should be framed for targeting habit-based interventions, to change knowledge and beliefs of patients.

\section{CONCLUSION}

Due to lack of standardized medication adherence metrics it is tough to make conclusions for consequences of medication non-adherence, barriers of adherence and rates (Gellad et al., 2011). Differences in terminology, study designs, definitions, and inclusion criteria have also been attributed to ineffectiveness of estimating adherence rates. Due to these, even the interventions that are used for improving adherence rates are not being able to get standardized (Conn et al., 2009). Techniques of data collection like pharmacy claims and 'use refill' may either overrate or underrate adherence based on inclusiveness of records. The method of pill counts may overrate non-adherence as patient may refill before the existing stock is finished. Vik et al., (2004) also presumes that those who report non-adherence are truly non-adherent while some patients who report adherence may not be. To capture the actual adherence rates one should use more than one measure of adherence.

Most studies confirm involvement of behavioural interventions to improve adherence (Conn et al., 2009) (Bain-Brickley et al., 2011). However, lost cost reminder devices do not improve adherence (Choudhry et al., 2017) (Kessler et al., 2018). The study of Spaan et al., (2018) found psychosocial interventions enhancing adherence among HIV patients. Data suggest that for improving adherence of patients who are under medication of more than six types of drugs, education is one of the best methods. Customized interventions like patient self-monitoring, patient education and stimuli to take medications could be developed on the basis of patient characteristics and type of non-adherence for effective adherence improvement (Haynes et al., 2008) (Conn et al., 2009) (Williams et al., 2008). Linn et al., (2011) conducted a systematic review of Internet-based adherence interventions and found results to be promising.

Non-adherence does not necessarily deteriorate patient health or cause surplus health care consumption (Alemagno et al., 2004) (Osterberg \& Blaschke, 2005). Certain studies report that if the treatment is inappropriate, flawless adherence to prescribed treatment can be harmful. The study of Launer et al., (2011) reports that "intensive glucose control increased mortality and did not prevent cardiovascular events in type 2 diabetes". It is difficult to measure adherence rates due to various types of biases (Hughes, 2007). Studies based on self-reports of non-adherence using survey method, may have different adherence rates due to the problems of patient recall bias and social desirability bias (Col et al., 1990).

Haynes et al., (2008) preformed a Cochrane review of randomized trials of adherence interventions. The results revealed that less than 50\% showed improvement in both clinical outcomes and long-term adherence. It was found that effective interventions were the ones, which had complex blends of numerous strategies. Individualized adherence tools have proved to be effective for adherence (Ostrop \& Gill, 2000) (Safren et al., 2003) (Nelson et al., 2018) (Vrijens et al., 2017). The study of T. S. 
Nguyen et al., (2019) reports that quality of life and medication adherence rate improved in patients after implementation of pharmaceutical care programme. The work of Jangi et al., (2018) reports that reminders sent for vaccines proved to be very effective. Calling intervention was cited as the most effective way of reminder system in the study. The use of pill bottle strip with toggles, digital timer cap and standard pillbox were cited as not effective. The study suggests sending targeted reminders and reminders in the form of videos and picture messages to enhance the medication adherence rates.

From the study conducted, it is clear that medication non-adherence is a common problem. It is necessary that patient, physician, service provider and family take adequate measures to ensure timely intake of medicines to expedite recovery and ensure full effect of medicine. Researchers believe that intervention of technology can help improve adherence rates but it must be used strategically so that the behaviour of patients can be targeted for improvement of adherence rates. Future research can be done to find out the effect of interventions specially targeting the behavioural models for addressing problems of non-adherence. There is lack of published literature for adherence of diet and exercise. This is a wide field of research, which can be explored for increasing the adherence of diet and exercise regimen.

\section{ACKNOWLEDGMENT}

This research was funded by All India Council for Technical Education (AICTE), India under Research Promotion Scheme for North Eastern Region (RPS-NER) for the grant entitled "Impact of Active Reminders on Medication Adherence" as per letter No 8-104/RIFD/RPS-NER/Policy-1/2019-19 dated 14 March 2019. 


\section{REFERENCES}

Adler, A. J., Martin, N., Mariani, J., Tajer, C. D., Owolabi, O. O., Free, C., Serrano, N. C., Casas, J. P., \& Perel, P. (2017). Mobile phone text messaging to improve medication adherence in secondary prevention of cardiovascular disease. Cochrane Database of Systematic Reviews. doi:10.1002/14651858.CD011851.pub2 PMID:28455948

Ahmed, I., Ahmad, N. S., Ali, S., Ali, S., George, A., Danish, H. S., \& Darzi, A. et al. (2018). Medication adherence apps: Review and content analysis. JMIR mHealth and uHealth, 6(3), e62. doi:10.2196/mhealth.6432 PMID:29549075

Aikens, J. E., Nease, D. E., Nau, D. P., Klinkman, M. S., \& Schwenk, T. L. (2005). Adherence to maintenancephase antidepressant medication as a function of patient beliefs about medication. Annals of Family Medicine, 3(1), 23-30. doi:10.1370/afm.238 PMID:15671187

Aikens, J. E., \& Piette, J. D. (2009). Diabetic patients' medication underuse, illness outcomes, and beliefs about antihyperglycemic and antihypertensive treatments. Diabetes Care, 32(1), 19-24. doi:10.2337/dc08-1533 PMID:18852334

Ajzen, I. (1991). The theory of planned behavior. Organizational Behavior and Human Decision Processes, 50(2), 179-211. doi:10.1016/0749-5978(91)90020-T

Albright, T. L., Parchman, M., \& Burge, S. K. et al. (2001). Predictors of self-care behavior in adults with type 2 diabetes: An RRNeST study. Family Medicine-Kansas City, 33(5), 354-360. PMID:11355645

Alemagno, S. A., Niles, S. A., \& Treiber, E. A. (2004). Using computers to reduce medication misuse of community-based seniors: Results of a pilot intervention program. Geriatric Nursing, 25(5), 281-285. doi:10.1016/j.gerinurse.2004.08.017 PMID:15486545

Amico, K. R., Mugavero, M., Krousel-Wood, M. A., Bosworth, H. B., \& Merlin, J. S. (2018). Advantages to Using Social-Behavioral Models of Medication Adherence in Research and Practice. Journal of General Internal Medicine, 33(2), 207-215. doi:10.1007/s11606-017-4197-5 PMID:29204968

Anderson, P. L., Glidden, D. V., Liu, A., Buchbinder, S., Lama, J. R., \& Guanira, J. V. (2012). Emtricitabinetenofovir concentrations and pre-exposure prophylaxis efficacy in men who have sex with men. Science Translational Medicine, 4(151).

Apter, A. J., Boston, R. C., George, M., Norfleet, A. L., Tenhave, T., Coyne, J. C., \& Feldman, H. I. et al. (2003). Modifiable barriers to adherence to inhaled steroids among adults with asthma: It's not just black and white. The Journal of Allergy and Clinical Immunology, 111(6), 1219-1226.

Apter, A. J., Reisine, S. T., Affleck, G., Barrows, E., \& ZuWallack, R. L. (1998). Adherence with twice-daily dosing of inhaled steroids: Socioeconomic and health-belief differences. American Journal of Respiratory and Critical Care Medicine, 157(6), 1810-1817. doi:10.1164/ajrccm.157.6.9712007 PMID:9620910

Arbuckle, C., Tomaszewski, D., Brown, L., Schommer, J., Morisky, D., Parlett-Pelleriti, C., \& Linstead, E. (2019). Exploring the relationship of digital information sources and medication adherence. Computers in Biology and Medicine, 109, 303-310. doi:10.1016/j.compbiomed.2019.04.023 PMID:31100583

Arnsten, J. H., Demas, P. A., Grant, R. W., Gourevitch, M. N., Farzadegan, H., Howard, A. A., \& Schoenbaum, E. E. (2002). Impact of active drug use on antiretroviral therapy adherence and viral suppression in HIV-infected drug users. Journal of General Internal Medicine, 17(5), 377-381. doi:10.1007/s11606-002-0044-3 PMID:12047736

Azar, M. M., Springer, S. A., Meyer, J. P., \& Altice, F. L. (2010). A systematic review of the impact of alcohol use disorders on HIV treatment outcomes, adherence to antiretroviral therapy and health care utilization. Drug and Alcohol Dependence, 112(3), 178-193. doi:10.1016/j.drugalcdep.2010.06.014 PMID:20705402

Badawy, S. M., Morrone, K., Thompson, A., \& Palermo, T. M. (2019). Computer and mobile technology interventions to promote medication adherence and disease management in people with thalassemia. Cochrane Database of Systematic Reviews. doi:10.1002/14651858.CD012900.pub2 PMID:31250923

Bain-Brickley, D., Butler, L. M., Kennedy, G. E., \& Rutherford, G. W. (2011). Interventions to improve adherence to antiretroviral therapy in children with HIV infection. Cochrane Database of Systematic Reviews, 12. doi:10.1002/14651858.CD009513 PMID:22161452 
Baker, Z. G. (2018). The Impact of Partnership Factors on Adherence to HIV Pre-Exposure Prophylaxis and Antiretroviral Therapy. UCLA.

Balkrishnan, R., Bhosle, M. J., Camacho, F. T., \& Anderson, R. T. (2006). Predictors of medication adherence and associated health care costs in an older population with overactive bladder syndrome: A longitudinal cohort study. The Journal of Urology, 175(3), 1067-1072. doi:10.1016/S0022-5347(05)00352-6 PMID:16469620

Balkrishnan, R., Rajagopalan, R., Camacho, F. T., Huston, S. A., Murray, F. T., \& Anderson, R. T. (2003). Predictors of medication adherence and associated health care costs in an older population with type 2 diabetes mellitus: A longitudinal cohort study. Clinical Therapeutics, 25(11), 2958-2971. doi:10.1016/S01492918(03)80347-8 PMID:14693318

Bandura, A. (1986). Social foundations of thought and action. Academic Press.

Banuelos Mota, A., Feliz Sala, E. E., Perdomo, J. M., Solis, J. A., Solorzano, W. M., Hochman, M., \& Reilly, J. M. (2019). Assessing Barriers to Medication Adherence Among Latinos with Diabetes: A Cross-sectional Study. Journal of General Internal Medicine. PMID:31161564

Bautista, L. E. (2008). Predictors of persistence with antihypertensive therapy: Results from the NHANES. American Journal of Hypertension, 21(2), 183-188. doi:10.1038/ajh.2007.33 PMID:18188161

Billups, S. J., Malone, D. C., \& Carter, B. L. (2000). The relationship between drug therapy noncompliance and patient characteristics, health-related quality of life, and health care costs. Pharmacotherapy: The Journal of Human Pharmacology and Drug Therapy, 20(8), 941-949. doi:10.1592/phco.20.11.941.35266 PMID:10939555

Britto, M. T., Munafo, J. K., Schoettker, P. J., Vockell, A.-L. B., Wimberg, J. A., \& Yi, M. S. (2012). Pilot and feasibility test of adolescent-controlled text messaging reminders. Clinical Pediatrics, 51(2), 114-121. doi:10.1177/0009922811412950 PMID:22019792

Brown, C., Battista, D. R., Bruehlman, R., Sereika, S. S., Thase, M. E., \& Dunbar-Jacob, J. (2005). Beliefs about antidepressant medications in primary care patients: Relationship to self-reported adherence. Medical Care, 43(12), 1203-1207. doi:10.1097/01.mlr.0000185733.30697.f6 PMID:16299431

Browne, S. H., Peloquin, C., Santillo, F., Haubrich, R., Muttera, L., Moser, K., Savage, G. M., Benson, C. A., $\&$ Blaschke, T. F. (2018). Digitizing Medicines for Remote Capture of Oral Medication Adherence Using Coencapsulation. Clinical Pharmacology and Therapeutics, 103(3), 502-510. doi:10.1002/cpt.760 PMID:28597911

Cafazzo, J. A., Casselman, M., Hamming, N., Katzman, D. K., \& Palmert, M. R. (2012). Design of an mHealth app for the self-management of adolescent type 1 diabetes: A pilot study. Journal of Medical Internet Research, 14(3), e70. doi:10.2196/jmir.2058 PMID:22564332

Capoccia, K., Odegard, P. S., \& Letassy, N. (2016). Medication Adherence With Diabetes Medication: A Systematic Review of the Literature. The Diabetes Educator, 42(1), 34-71. doi:10.1177/0145721715619038 PMID:26637240

Chambers, C. V., Markson, L., Diamond, J. J., Lasch, L., \& Berger, M. (1999). Health beliefs and compliance with inhaled corticosteroids by asthmatic patients in primary care practices. Respiratory Medicine, 93(2), 88-94. doi:10.1016/S0954-6111(99)90296-2 PMID:10464858

Chander, G., Lau, B., \& Moore, R. D. (2006). Hazardous alcohol use: a risk factor for non-adherence and lack of suppression in HIV infection. Journal of Acquired Immune Deficiency Syndromes, 43(4), 411.

Chang, L. W., Kagaayi, J., Nakigozi, G., Packer, A. H., Serwadda, D., Quinn, T. C., . . Reynolds, S. J. (2008). Letter to the Editor: Responding to the Human Resource Crisis: Peer Health Workers, Mobile Phones, and HIV Care in Rakai, Uganda. AIDS Patient Care and STDs, 22(3), 173-174.

Chao, J., Nau, D. P., \& Aikens, J. E. (2007). Patient-reported perceptions of side effects of antihyperglycemic medication and adherence to medication regimens in persons with diabetes mellitus. Clinical Therapeutics, 29(1), 177-180. doi:10.1016/j.clinthera.2007.01.014 PMID:17379058

Chapman, R. H., Benner, J. S., Petrilla, A. A., Tierce, J. C., Collins, S. R., Battleman, D. S., \& Schwartz, J. S. (2005). Predictors of adherence with antihypertensive and lipid-lowering therapy. Archives of Internal Medicine, 165(10), 1147-1152. doi:10.1001/archinte.165.10.1147 PMID:15911728 
Choudhry, N. K., Krumme, A. A., Ercole, P. M., Girdish, C., Tong, A. Y., Khan, N. F., Brennan, T. A., Matlin, O. S., Shrank, W. H., \& Franklin, J. M. (2017). Effect of reminder devices on medication adherence: The REMIND randomized clinical trial. JAMA Internal Medicine, 177(5), 624-631. doi:10.1001/jamainternmed.2016.9627 PMID:28241271

Clark, N. M., \& Dodge, J. A. (1999). Exploring self-efficacy as a predictor of disease management. Health Education \& Behavior, 26(1), 72-89. doi:10.1177/109019819902600107 PMID:9952053

Claxton, A. J., Cramer, J., \& Pierce, C. (2001). A systematic review of the associations between dose regimens and medication compliance. Clinical Therapeutics, 23(8), 1296-1310. doi:10.1016/S0149-2918(01)80109-0 PMID:11558866

Col, N., Fanale, J. E., \& Kronholm, P. (1990). The role of medication noncompliance and adverse drug reactions in hospitalizations of the elderly. Archives of Internal Medicine, 150(4), 841-845. doi:10.1001/ archinte.1990.00390160093019 PMID:2327844

Conn, V. S., Hafdahl, A. R., Cooper, P. S., Ruppar, T. M., Mehr, D. R., \& Russell, C. L. (2009). Interventions to improve medication adherence among older adults: Meta-analysis of adherence outcomes among randomized controlled trials. The Gerontologist, 49(4), 447-462. doi:10.1093/geront/gnp037 PMID:19460887

Conn, V. S., \& Ruppar, T. M. (2017). Medication adherence outcomes of 771 intervention trials: Systematic review and meta-analysis. Preventive Medicine, 99, 269-276. doi:10.1016/j.ypmed.2017.03.008 PMID:28315760

Conn, V. S., Ruppar, T. M., Enriquez, M., \& Cooper, P. (2016). Medication adherence interventions that target subjects with adherence problems: Systematic review and meta-analysis. Research in Social \& Administrative Pharmacy, 12(2), 218-246. doi:10.1016/j.sapharm.2015.06.001 PMID:26164400

Cook, R. L., Sereika, S. M., Hunt, S. C., Woodward, W. C., Erlen, J. A., \& Conigliaro, J. (2001). Problem drinking and medication adherence among persons with HIV infection. Journal of General Internal Medicine, 16(2), 83-88. doi:10.1111/j.1525-1497.2001.00122.x PMID:11251758

Corneli, A. L., Deese, J., Wang, M., Taylor, D., Ahmed, K., Agot, K., Lombaard, J., Manongi, R., Kapiga, S., Kashuba, A., \& Van Damme, L. (1999). others. (2014). FEM-PrEP: Adherence patterns and factors associated with adherence to a daily oral study product for pre-exposure prophylaxis. Journal of Acquired Immune Deficiency Syndromes, 66(3), 324-331. doi:10.1097/QAI.0000000000000158 PMID:25157647

Cramer, J. A., Mattson, R. H., Prevey, M. L., Scheyer, R. D., \& Ouellette, V. L. (1989). How often is medication taken as prescribed?: A novel assessment technique. Journal of the American Medical Association, 261(22), 3273-3277. doi:10.1001/jama.1989.03420220087032 PMID:2716163

Cramer, J. A., Roy, A., Burrell, A., Fairchild, C. J., Fuldeore, M. J., Ollendorf, D. A., \& Wong, P. K. (2008). Medication compliance and persistence: Terminology and definitions. Value in Health, 11(1), 44-47. doi:10.1111/ j.1524-4733.2007.00213.x PMID:18237359

Cukor, D., Rosenthal, D. S., Jindal, R. M., Brown, C. D., \& Kimmel, P. L. (2009). Depression is an important contributor to low medication adherence in hemodialyzed patients and transplant recipients. Kidney International, 75(11), 1223-1229. doi:10.1038/ki.2009.51 PMID:19242502

Cutler, R. L., Fernandez-Llimos, F., Frommer, M., Benrimoj, C., \& Garcia-Cardenas, V. (2018). Economic impact of medication non-adherence by disease groups: A systematic review. BMJ Open, 8(1), e016982. doi:10.1136/ bmjopen-2017-016982 PMID:29358417

Daleboudt, G. M. N., Broadbent, E., McQueen, F., \& Kaptein, A. A. (2011). Intentional and unintentional treatment nonadherence in patients with systemic lupus erythematosus. Arthritis Care and Research, 63(3), 342-350. PMID:21120967

Dayer, L., Heldenbrand, S., Anderson, P., Gubbins, P. O., \& Martin, B. C. (2013). Smartphone medication adherence apps: Potential benefits to patients and providers. Journal of the American Pharmacists Association, 53(2), 172-181. doi:10.1331/JAPhA.2013.12202 PMID:23571625

De Geest, S., Zullig, L. L., Dunbar-Jacob, J., Hughes, D., Wilson, I. B., \& Vrijens, B. (2019). Improving medication adherence research reporting: European society for patient adherence, compliance and persistence medication adherence reporting guideline. The Journal of Cardiovascular Nursing, 34(3), 199-200. doi:10.1097/ JCN.0000000000000572 PMID:30973491 
De Smet, B. D., Erickson, S. R., \& Kirking, D. M. (2006). Self-reported adherence in patients with asthma. The Annals of Pharmacotherapy, 40(3), 414-420. doi:10.1345/aph.1G475 PMID:16507619

Delpier, T., Giordana, S., \& Wedin, B. M. (2013). Decreasing sugar-sweetened beverage consumption in the rural adolescent population. Journal of Pediatric Health Care, 27(6), 470-478. doi:10.1016/j.pedhc.2012.07.002 PMID:22932228

Dew, M. A., DiMartini, A. F., Dabbs, A. D. V., Zomak, R., De Geest, S., \& Dobbels, F. et al.. (2008). Adherence to the medical regimen during the first two years after lung transplantation. Transplantation, 85(2), 193-202. doi:10.1097/TP.0b013e318160135f PMID:18212623

DiMatteo, M. R. (2004). Variations in patients' adherence to medical recommendations: A quantitative review of 50 years of research. Medical Care, 42(3), 200-209. doi:10.1097/01.mlr.0000114908.90348.f9 PMID:15076819

DiMatteo, M. R., Haskard-Zolnierek, K. B., \& Martin, L. R. (2012). Improving patient adherence: A threefactor model to guide practice. Health Psychology Review, 6(1), 74-91. doi:10.1080/17437199.2010.537592

Doggrell, S. A. (2010). Adherence to medicines in the older-aged with chronic conditions. Drugs \& Aging, 27(3), 239-254. doi:10.2165/11532870-000000000-00000 PMID:20210369

Esposito, D., Bagchi, A. D., Verdier, J. M., Bencio, D. S., \& Kim, M. S. (2009). Medicaid beneficiaries with congestive heart failure: Association of medication adherence with healthcare use and costs. The American Journal of Managed Care, 15(7), 437-445. PMID:19589011

Faught, R. E., Weiner, J. R., Guérin, A., Cunnington, M. C., \& Duh, M. S. (2009). Impact of nonadherence to antiepileptic drugs on health care utilization and costs: Findings from the RANSOM study. Epilepsia, 50(3), 501-509. doi:10.1111/j.1528-1167.2008.01794.x PMID:19183224

Fenerty, S. D., West, C., Davis, S. A., Kaplan, S. G., \& Feldman, S. R. (2012). The effect of reminder systems on patients' adherence to treatment. Patient Preference and Adherence, 6, 127. PMID:22379363

Fernandez-Lazaro, C. I., Adams, D. P., Fernandez-Lazaro, D., Garcia-González, J. M., Caballero-Garcia, A., \& Miron-Canelo, J. A. (2019). Medication adherence and barriers among low-income, uninsured patients with multiple chronic conditions. Research in Social \& Administrative Pharmacy, 15(6), 744-753. doi:10.1016/j. sapharm.2018.09.006 PMID:30241872

Ficke, D. L., \& Farris, K. B. (2005). Use of the transtheoretical model in the medication use process. The Annals of Pharmacotherapy, 39(7-8), 1325-1330. doi:10.1345/aph.1G122 PMID:15956227

Fjeldsoe, B. S., Marshall, A. L., \& Miller, Y. D. (2009). Behavior change interventions delivered by mobile telephone short-message service. American Journal of Preventive Medicine, 36(2), 165-173. doi:10.1016/j. amepre.2008.09.040 PMID:19135907

Frick, P. A., Lavreys, L., Mandaliya, K., \& Kreiss, J. K. (2001). Impact of an alarm device on medication compliance in women in Mombasa, Kenya. International Journal of STD \& AIDS, 12(5), 329-333. doi:10.1258/0956462011923048 PMID:11368808

Friedman, D. S., Okeke, C. O., Jampel, H. D., Ying, G., Plyler, R. J., Jiang, Y., \& Quigley, H. A. (2009). Risk factors for poor adherence to eyedrops in electronically monitored patients with glaucoma. Ophthalmology, 116(6), 1097-1105. doi:10.1016/j.ophtha.2009.01.021 PMID:19376591

Gadkari, A. S., \& McHorney, C. A. (2012). Unintentional non-adherence to chronic prescription medications: How unintentional is it really? BMC Health Services Research, 12(1), 98. doi:10.1186/1472-6963-12-98 PMID:22510235

Galloway, G. P., Coyle, J. R., Guillén, J. E., Flower, K., \& Mendelson, J. E. (2011). A simple, novel method for assessing medication adherence: Capsule photographs taken with cellular telephones. Journal of Addiction Medicine, 5(3), 170-174. doi:10.1097/ADM.0b013e3181fcb5fd PMID:21844832

Gamble, K. H. (2009). Beyond phones. With the proper infrastructure, smartphones can help improve clinician satisfaction and increase EMR use. Healthcare Informatics: The Business Magazine for Information and Communication Systems, 26(8), 23-24. PMID:19722325 
Garfield, S., Clifford, S., Eliasson, L., Barber, N., \& Willson, A. (2011). Suitability of measures of self-reported medication adherence for routine clinical use: A systematic review. BMC Medical Research Methodology, 11(1), 149. doi:10.1186/1471-2288-11-149 PMID:22050830

Gatti, M. E., Jacobson, K. L., Gazmararian, J. A., Schmotzer, B., \& Kripalani, S. (2009). Relationships between beliefs about medications and adherence. American Journal of Health-System Pharmacy, 66(7), 657-664. doi:10.2146/ajhp080064 PMID:19299373

Gazmararian, J. A., Kripalani, S., Miller, M. J., Echt, K. V., Ren, J., \& Rask, K. (2006). Factors associated with medication refill adherence in cardiovascular-related diseases. Journal of General Internal Medicine, 21(12), 1215-1221. doi:10.1111/j.1525-1497.2006.00591.x PMID:17105519

Gehi, A., Haas, D., Pipkin, S., \& Whooley, M. A. (2005). Depression and medication adherence in outpatients with coronary heart disease: Findings from the Heart and Soul Study. Archives of Internal Medicine, 165(21), 2508-2513. doi:10.1001/archinte.165.21.2508 PMID:16314548

Gellad, W. F., Grenard, J. L., \& Marcum, Z. A. (2011). A systematic review of barriers to medication adherence in the elderly: Looking beyond cost and regimen complexity. The American Journal of Geriatric Pharmacotherapy, 9(1), 11-23. doi:10.1016/j.amjopharm.2011.02.004 PMID:21459305

George, J., Elliott, R. A., \& Stewart, D. C. (2008). A systematic review of interventions to improve medication taking in elderly patients prescribed multiple medications. Drugs \& Aging, 25(4), 307-324. doi:10.2165/00002512200825040-00004 PMID:18361541

Gonzalez, J., Safren, S. A., Delahanty, L. M., Cagliero, E., Wexler, D. J., Meigs, J. B., \& Grant, R. W. (2008). Symptoms of depression prospectively predict poorer self-care in patients with Type 2 diabetes. Diabetic Medicine, 25(9), 1102-1107. doi:10.1111/j.1464-5491.2008.02535.x PMID:19183315

Gonzalez, J. S., Safren, S. A., Cagliero, E., Wexler, D. J., Delahanty, L., Wittenberg, E., Blais, M. A., Meigs, J. B., \& Grant, R. W. (2007). Depression, self-care, and medication adherence in type 2 diabetes: Relationships across the full range of symptom severity. Diabetes Care, 30(9), 2222-2227. doi:10.2337/dc07-0158 PMID:17536067

Graves, M. M., Roberts, M. C., Rapoff, M., \& Boyer, A. (2009). The efficacy of adherence interventions for chronically ill children: A meta-analytic review. Journal of Pediatric Psychology, 35(4), 368-382. doi:10.1093/ jpepsy/jsp072 PMID:19710248

Hajjar, E. R., Cafiero, A. C., \& Hanlon, J. T. (2007). Polypharmacy in elderly patients. The American Journal of Geriatric Pharmacotherapy, 5(4), 345-351. doi:10.1016/j.amjopharm.2007.12.002 PMID:18179993

Harbig, P., Barat, I., \& Damsgaard, E. M. (2012). Suitability of an electronic reminder device for measuring drug adherence in elderly patients with complex medication. Journal of Telemedicine and Telecare, 18(6), 352-356. doi:10.1258/jtt.2012.120120 PMID:22912488

Haynes, R. (1996). Systematic review of randomised trials of interventions to assist patients to follow prescriptions for medications. Lancet, 348(9024), 383-386. doi:10.1016/S0140-6736(96)01073-2 PMID:8709739

Haynes, R. B., Ackloo, E., Sahota, N., McDonald, H. P., \& Yao, X. (2008). Interventions for enhancing adherence to prescribed medications. Cochrane Database of Systematic Reviews, 2.

Henny, K. D., Wilkes, A. L., McDonald, C. M., Denson, D. J., \& Neumann, M. S. (2018). A Rapid Review of eHealth Interventions Addressing the Continuum of HIV Care (2007-2017). AIDS and Behavior, 22(1), 43-63. doi:10.1007/s10461-017-1923-2 PMID:28983684

Hepke, K. L., Martus, M. T., \& Share, D. A. (2004). Costs and utilization associated with pharmaceutical adherence in a diabetic population. The American Journal of Managed Care, $10(2 \mathrm{Pt} 2), 144-151$. PMID:15005507

Hinkin, C. H., Hardy, D. J., Mason, K. I., Castellon, S. A., Durvasula, R. S., Lam, M. N., \& Stefaniak, M. (2004). Medication adherence in HIV-infected adults: Effect of patient age, cognitive status, and substance abuse. AIDS (London, England), 18(Suppl 1), S19. doi:10.1097/00002030-200401001-00004 PMID:15075494

Ho, P. M., Rumsfeld, J. S., Masoudi, F. A., McClure, D. L., Plomondon, M. E., Steiner, J. F., \& Magid, D. J. (2006). Effect of medication nonadherence on hospitalization and mortality among patients with diabetes mellitus. Archives of Internal Medicine, 166(17), 1836-1841. doi:10.1001/archinte.166.17.1836 PMID:17000939

Holloway, K., \& Van Dijk, L. (2011). The world medicines situation 2011. Rational use of medicines. WHO. 
Holt, E., Joyce, C., Dornelles, A., Morisky, D., Webber, L. S., Muntner, P., \& Krousel-Wood, M. (2013). Sex differences in barriers to antihypertensive medication adherence: Findings from the cohort study of medication adherence among older adults. Journal of the American Geriatrics Society, 61(4), 558-564. doi:10.1111/ jgs.12171 PMID:23528003

Hughes, D. (2007). When drugs don't work. PharmacoEconomics, 25(8), 621-635. doi:10.2165/00019053200725080-00001 PMID:17640105

Hyre, A. D., Krousel-Wood, M. A., Muntner, P., Kawasaki, L., \& DeSalvo, K. B. (2007). Prevalence and predictors of poor antihypertensive medication adherence in an urban health clinic setting. Journal of Clinical Hypertension, 9(3), 179-186. doi:10.1111/j.1524-6175.2007.06372.x PMID:17344769

Insel, K., Morrow, D., Brewer, B., \& Figueredo, A. (2006). Executive function, working memory, and medication adherence among older adults. The Journals of Gerontology. Series B, Psychological Sciences and Social Sciences, 61(2), 102-P107. doi:10.1093/geronb/61.2.P102 PMID:16497953

Jangi, M., Ferandez-de-las-Penas, C., Tara, M., Moghbeli, F., Ghaderi, F., \& Javanshir, K. (2018). A systematic review on reminder systems in physical therapy. Caspian Journal of Internal Medicine, 9(1), 7-15. PMID:29387313

Janson, S. L., Earnest, G., Wong, K. P., \& Blanc, P. D. (2008). Predictors of asthma medication nonadherence. Heart \& Lung: The Journal of Acute and Critical Care, 37(3), 211-218. doi:10.1016/j.hrtlng.2007.05.014 PMID:18482633

Kaplan, R. C., Bhalodkar, N. C., Brown, E. J. Jr, White, J., \& Brown, D. L. (2004). Race, ethnicity, and sociocultural characteristics predict noncompliance with lipid-lowering medications. Preventive Medicine, 39(6), 1249-1255. doi:10.1016/j.ypmed.2004.04.041 PMID:15539064

Kerr, D. A., Pollard, C. M., Howat, P., Delp, E. J., Pickering, M., Kerr, K. R., Dhaliwal, S. S., Pratt, I. S., Wright, J., \& Boushey, C. J. (2012). Connecting Health and Technology (CHAT): Protocol of a randomized controlled trial to improve nutrition behaviours using mobile devices and tailored text messaging in young adults. $B M C$ Public Health, 12(1), 477. doi:10.1186/1471-2458-12-477 PMID:22726532

Kessler, J. B., Troxel, A. B., Asch, D. A., Mehta, S. J., Marcus, N., Lim, R., Zhu, J., Shrank, W., Brennan, T., \& Volpp, K. G. (2018). Partners and Alerts in Medication Adherence: A Randomized Clinical Trial. Journal of General Internal Medicine, 33(9), 1536-1542. doi:10.1007/s11606-018-4389-7 PMID:29546659

Khanderia, U., Townsend, K. A., Erickson, S. R., Vlasnik, J., Prager, R. L., \& Eagle, K. A. (2008). Medication adherence following coronary artery bypass graft surgery: Assessment of beliefs and attitudes. The Annals of Pharmacotherapy, 42(2), 192-199. doi:10.1345/aph.1K497 PMID:18198242

Kharrazi, H., Chisholm, R., VanNasdale, D., \& Thompson, B. (2012). Mobile personal health records: An evaluation of features and functionality. International Journal of Medical Informatics, 81(9), 579-593. doi:10.1016/j.jimedinf.2012.04.007 PMID:22809779

Kilbourne, A. M., Reynolds, C. F. III, Good, C. B., Sereika, S. M., Justice, A. C., \& Fine, M. J. (2005). How does depression influence diabetes medication adherence in older patients? The American Journal of Geriatric Psychiatry, 13(3), 202-210. doi:10.1097/00019442-200503000-00005 PMID:15728751

Kim, E.-Y., Han, H.-R., Jeong, S., Kim, K. B., Park, H., Kang, E., Shin, H. S., \& Kim, M. T. (2007). Does knowledge matter?: Intentional medication nonadherence among middle-aged Korean Americans with high blood pressure. The Journal of Cardiovascular Nursing, 22(5), 397-404. doi:10.1097/01.JCN.0000287038.23186.bd PMID: 17724422

Kravitz, R. L., \& Melnikow, J. (2004). Medical adherence research: time for a change in direction? LWW.

Kulkarni, A. S., Balkrishnan, R., Anderson, R. T., Edin, H. M., Kirsch, J., \& Stacy, M. A. (2008). Medication adherence and associated outcomes in medicare health maintenance organization-enrolled older adults with Parkinson's disease. Movement Disorders, 23(3), 359-365. doi:10.1002/mds.21831 PMID:18074366

Lau, D. T., \& Nau, D. P. (2004). Oral antihyperglycemic medication nonadherence and subsequent hospitalization among individuals with type 2 diabetes. Diabetes Care, 27(9), 2149-2153. doi:10.2337/diacare.27.9.2149 PMID:15333476 
Launer, L. J., Miller, M. E., Williamson, J. D., Lazar, R. M., Gerstein, H. C., Murray, A. M., Sullivan, M., Horowitz, K. R., Ding, J., Marcovina, S., Lovato, L. C., Lovato, J., Margolis, K. L., O’Connor, P., Lipkin, E. W., Hirsch, J., Coker, L., Maldjian, J., Sunshine, J. L., \& Bryan, R. N. et al. (2011). Effects of intensive glucose lowering on brain structure and function in people with type 2 diabetes (ACCORD MIND): A randomised openlabel substudy. Lancet Neurology, 10(11), 969-977. doi:10.1016/S1474-4422(11)70188-0 PMID:21958949

Le, T. T., Bilderback, A., Bender, B., Wamboldt, F. S., Turner, C. F., Rand, C. S., \& Bartlett, S. J. (2008). Do asthma medication beliefs mediate the relationship between minority status and adherence to therapy? The Journal of Asthma, 45(1), 33-37. doi:10.1080/02770900701815552 PMID:18259993

Lee, D.-S., Jeon, B. G., Ihm, C., Park, J.-K., \& Jung, M. Y. (2011). A simple and smart telemedicine device for developing regions: A pocket-sized colorimetric reader. Lab on a Chip, 11(1), 120-126. doi:10.1039/ C0LC00209G PMID:21109898

Lehane, E., \& McCarthy, G. (2007). Intentional and unintentional medication non-adherence: A comprehensive framework for clinical research and practice? A discussion paper. International Journal of Nursing Studies, 44(8), 1468-1477. doi:10.1016/j.ijnurstu.2006.07.010 PMID:16973166

Lester, R., \& Karanja, S. (2008). Mobile phones: Exceptional tools for HIV/AIDS, health, and crisis management. The Lancet. Infectious Diseases, 8(12), 738-739. doi:10.1016/S1473-3099(08)70265-2 PMID:19022188

Li, W.-W., \& Froelicher, E. S. (2007). Gender differences in Chinese immigrants: Predictors for antihypertensive medication adherence. Journal of Transcultural Nursing, 18(4), 331-338. doi:10.1177/1043659607305194 PMID:17911573

Li, W.-W., Stewart, A. L., Stotts, N., \& Froelicher, E. S. (2006). Cultural factors associated with antihypertensive medication adherence in Chinese immigrants. The Journal of Cardiovascular Nursing, 21(5), 354-362. doi:10.1097/00005082-200609000-00005 PMID:16966912

Lin, E. H. B., Katon, W., Von Korff, M., Rutter, C., Simon, G. E., Oliver, M., Ciechanowski, P., Ludman, E. J., Bush, T., \& Young, B. (2004). Relationship of depression and diabetes self-care, medication adherence, and preventive care. Diabetes Care, 27(9), 2154-2160. doi:10.2337/diacare.27.9.2154 PMID:15333477

Linn, A. J., Vervloet, M., van Dijk, L., Smit, E. G., \& Van Weert, J. C. M. (2011). Effects of eHealth interventions on medication adherence: A systematic review of the literature. Journal of Medical Internet Research, 13(4), e103. doi:10.2196/jmir.1738 PMID:22138112

Maddison, R., Stewart, R., Doughty, R., Scott, T., Kerr, A., Benatar, J., Whittaker, R., Rawstorn, J. C., Rolleston, A., Jiang, Y., Estabrooks, P., Sullivan, R. K., Bartley, H., \& Pfaeffli Dale, L. (2018). Text4Heart II - improving medication adherence in people with heart disease: A study protocol for a randomized controlled trial. Trials, 19(1), 70. doi:10.1186/s13063-018-2468-z PMID:29370829

Mahtani, K. R., Heneghan, C. J., Glasziou, P. P., \& Perera, R. (2011). Reminder packaging for improving adherence to self-administered long-term medications. Cochrane Database of Systematic Reviews, 9. doi:10.1002/14651858. CD005025.pub3 PMID:21901694

Malhotra, S., Karan, R. S., Pandhi, P., \& Jain, S. (2001). Drug related medical emergencies in the elderly: Role of adverse drug reactions and non-compliance. Postgraduate Medical Journal, 77(913), 703-707. doi:10.1136/ pmj.77.913.703 PMID:11677279

Mann, D. M., Allegrante, J. P., Natarajan, S., Halm, E. A., \& Charlson, M. (2007). Predictors of adherence to statins for primary prevention. Cardiovascular Drugs and Therapy, 21(4), 311-316. doi:10.1007/s10557-0076040-4 PMID:17665294

Mann, D. M., Ponieman, D., Leventhal, H., \& Halm, E. A. (2009). Predictors of adherence to diabetes medications: The role of disease and medication beliefs. Journal of Behavioral Medicine, 32(3), 278-284. doi:10.1007/ s10865-009-9202-y PMID:19184390

Marrazzo, J. M., Ramjee, G., Richardson, B. A., Gomez, K., Mgodi, N., Nair, G., Palanee, T., Nakabiito, C., van der Straten, A., Noguchi, L., Hendrix, C. W., Dai, J. Y., Ganesh, S., Mkhize, B., Taljaard, M., Parikh, U. M., Piper, J., Mâsse, B., Grossman, C., \& Chirenje, Z. M. et al. (2015). Tenofovir-based preexposure prophylaxis for HIV infection among African women. The New England Journal of Medicine, 372(6), 509-518. doi:10.1056/ NEJMoa1402269 PMID:25651245 
McHorney, C. A., Schousboe, J. T., Cline, R. R., \& Weiss, T. W. (2007). The impact of osteoporosis medication beliefs and side-effect experiences on non-adherence to oral bisphosphonates. Current Medical Research and Opinion, 23(12), 3137-3152. doi:10.1185/030079907X242890 PMID:17988435

Mochari, H., Ferris, A., Adigopula, S., Henry, G., \& Mosca, L. (2007). Cardiovascular disease knowledge, medication adherence, and barriers to preventive action in a minority population. Preventive Cardiology, 10(4), 190-195. doi:10.1111/j.1520-037X.2007.06619.x PMID:17917515

Mondesir, F. L., Carson, A. P., Durant, R. W., Lewis, M. W., Safford, M. M., \& Levitan, E. B. (2018). Association of functional and structural social support with medication adherence among individuals treated for coronary heart disease risk factors: Findings from the REasons for Geographic and Racial Differences in Stroke (REGARDS) study. PLoS One, 13(6), e0198578. doi:10.1371/journal.pone.0198578 PMID:29949589

Morawski, K., Ghazinouri, R., Krumme, A., Lauffenburger, J. C., Lu, Z., Durfee, E., Oley, L., Lee, J., Mohta, N., Haff, N., Juusola, J. L., \& Choudhry, N. K. (2018). Association of a smartphone application with medication adherence and blood pressure control: The MedISAFE-BP randomized clinical trial. JAMA Internal Medicine, 178(6), 802-809. doi:10.1001/jamainternmed.2018.0447 PMID:29710289

Mrosek, R., Dehling, T., \& Sunyaev, A. (2015). Taxonomy of health IT and medication adherence. Health Policy and Technology, 4(3), 215-224. doi:10.1016/j.hlpt.2015.04.003

Náfrádi, L., Nakamoto, K., \& Schulz, P. J. (2017). Is patient empowerment the key to promote adherence? A systematic review of the relationship between self-efficacy, health locus of control and medication adherence. PLoS One, 12(10), e0186458. doi:10.1371/journal.pone.0186458 PMID:29040335

Nair, K. V., Belletti, D. A., Doyle, J. J., Allen, R. R., McQueen, R. B., Saseen, J. J., \& Jan, S. et al. (2011). Understanding barriers to medication adherence in the hypertensive population by evaluating responses to a telephone survey. Patient Preference and Adherence, 5, 195. doi:10.2147/PPA.S18481 PMID:21573051

Nau, D. P., Aikens, J. E., \& Pacholski, A. M. (2007). Effects of gender and depression on oral medication adherence in persons with type 2 diabetes mellitus. Gender Medicine, 4(3), 205-213. doi:10.1016/S15508579(07)80041-6 PMID:18022588

Nelson, L. A., Wallston, K. A., Kripalani, S., LeStourgeon, L. M., Williamson, S. E., \& Mayberry, L. S. (2018). Assessing barriers to diabetes medication adherence using the Information-Motivation-Behavioral skills model. Diabetes Research and Clinical Practice, 142, 374-384. doi:10.1016/j.diabres.2018.05.046 PMID:29879495

Nguyen, G. C., LaVeist, T. A., Harris, M. L., Datta, L. W., Bayless, T. M., \& Brant, S. R. (2009). Patient trustin-physician and race are predictors of adherence to medical management in inflammatory bowel disease. Inflammatory Bowel Diseases, 15(8), 1233-1239. doi:10.1002/ibd.20883 PMID:19177509

Nguyen, T. S., Nguyen, T. L. H., Van Pham, T. T., Hua, S., Ngo, Q. C., \& Li, S. C. (2019). Impact of pharmaceutical care in the improvement of medication adherence and quality of life for COPD patients in Vietnam. Respiratory Medicine, 153, 31-37. doi:10.1016/j.rmed.2019.05.006 PMID:31136931

Noh, J.-H., Cho, Y.-J., Nam, H.-W., Kim, J.-H., Kim, D.-J., Yoo, H.-S., Kwon, Y.-W., Woo, M.-H., Cho, J.-W., Hong, M.-H., Yoo, J.-H., Gu, M.-J., Kim, S.-A., An, K.-E., Jang, S.-M., Kim, E.-K., \& Yoo, H.-J. (2010). Webbased comprehensive information system for self-management of diabetes mellitus. Diabetes Technology \& Therapeutics, 12(5), 333-337. doi:10.1089/dia.2009.0122 PMID:20388042

O'grady, M. J., Retterath, A. J., Keenan, D. B., Kurtz, N., Cantwell, M., Spital, G., Kremliovsky, M. N., Roy, A., Davis, E. A., Jones, T. W., \& Ly, T. T. (2012). The use of an automated, portable glucose control system for overnight glucose control in adolescents and young adults with type 1 diabetes. Diabetes Care, 35(11), 2182-2187. doi:10.2337/dc12-0761 PMID:22875230

Ogedegbe, G., Chaplin, W., Schoenthaler, A., Statman, D., Berger, D., Richardson, T., Phillips, E., Spencer, J., \& Allegrante, J. P. (2008). A practice-based trial of motivational interviewing and adherence in hypertensive African Americans. American Journal of Hypertension, 21(10), 1137-1143. doi:10.1038/ajh.2008.240 PMID:18654123

Oh, C.-K., Bang, J. B., Kim, S.-J., Huh, K. H., Kim, S. J., Jeon, J. S., \& Kim, Y. S. et al. (2019). Improvement of medication adherence with simplified once-daily immunosuppressive regimen in stable kidney transplant recipients: A prospective cohort study. Asian Journal of Surgery. PMID:31353239 
Osterberg, L., \& Blaschke, T. (2005). Adherence to medication. The New England Journal of Medicine, 353(5), 487-497. doi:10.1056/NEJMra050100 PMID:16079372

Ostrop, N. J., \& Gill, M. J. (2000). Antiretroviral medication adherence and persistence with respect to adherence tool usage. AIDS Patient Care and STDs, 14(7), 351-358. doi:10.1089/108729100413220 PMID:10935052

Oyugi, J. H., Byakika-Tusiime, J., Ragland, K., Laeyendecker, O., Mugerwa, R., Kityo, C., Mugyenyi, P., Quinn, T. C., \& Bangsberg, D. R. (2007). Treatment interruptions predict resistance in HIV-positive individuals purchasing fixed-dose combination antiretroviral therapy in Kampala, Uganda. AIDS (London, England), 21(8), 965-971. doi:10.1097/QAD.0b013e32802e6bfa PMID:17457090

Patel, R. P., \& Taylor, S. D. (2002). Factors affecting medication adherence in hypertensive patients. The Annals of Pharmacotherapy, 36(1), 40-45. doi:10.1345/aph.1A046 PMID:11816255

Paterson, D. L., Potoski, B., \& Capitano, B. (2002). Measurement of adherence to antiretroviral medications. Journal of Acquired Immune Deficiency Syndromes, 31, S103-6.

Pedan, A., Varasteh, L. T., \& Schneeweiss, S. (2007). Analysis of factors associated with statin adherence in a hierarchical model considering physician, pharmacy, patient, and prescription characteristics. Journal of Managed Care Pharmacy, 13(6), 487-496. doi:10.18553/jmcp.2007.13.6.487 PMID:17672810

Petrie, K. J., \& Weinman, J. (1997). Perceptions of health and illness: Current research and applications. Taylor $\&$ Francis.

Phatak, H. M., \& Thomas, J. III. (2006). Relationships between beliefs about medications and nonadherence to prescribed chronic medications. The Annals of Pharmacotherapy, 40(10), 1737-1742. doi:10.1345/aph.1H153 PMID: 16985088

Platt, A. B., Localio, A. R., Brensinger, C. M., Cruess, D. G., Christie, J. D., Gross, R., Parker, C. S., Price, M., Metlay, J. P., Cohen, A., Newcomb, C. W., Strom, B. L., Laskin, M. S., \& Kimmel, S. E. (2008). Risk factors for nonadherence to warfarin: Results from the IN-RANGE study. Pharmacoepidemiology and Drug Safety, 17(9), 853-860. doi:10.1002/pds.1556 PMID:18271059

Polonsky, W. H., \& Henry, R. R. (2016). Poor medication adherence in type 2 diabetes: Recognizing the scope of the problem and its key contributors. Patient Preference and Adherence, 10, 1299-1306. doi:10.2147/PPA. S106821 PMID:27524885

Pop-Eleches, C., Thirumurthy, H., Habyarimana, J. P., Zivin, J. G., Goldstein, M. P., De Walque, D., MacKeen, L., Haberer, J., Kimaiyo, S., Sidle, J., Ngare, D., \& Bangsberg, D. R. (2011). Mobile phone technologies improve adherence to antiretroviral treatment in a resource-limited setting: A randomized controlled trial of text message reminders. AIDS (London, England), 25(6), 825-834. doi:10.1097/QAD.0b013e32834380c1 PMID:21252632

Protopopescu, C., Raffi, F., Roux, P., Reynes, J., Dellamonica, P., Spire, B., Leport, C., \& Carrieri, M.-P. (2009). Factors associated with non-adherence to long-term highly active antiretroviral therapy: A 10 year follow-up analysis with correction for the bias induced by missing data. The Journal of Antimicrobial Chemotherapy, 64(3), 599-606. doi:10.1093/jac/dkp232 PMID:19602563

Roebuck, M. C., Liberman, J. N., Gemmill-Toyama, M., \& Brennan, T. A. (2011). Medication adherence leads to lower health care use and costs despite increased drug spending. Health Affairs, 30(1), 91-99. doi:10.1377/ hlthaff.2009.1087 PMID:21209444

Rolnick, S., Pawloski, P., Bruzek, R., Hedblom, B., Asche, S., Fustgaard, M., \& Meier, D. (2011). PS2-32: Barriers and facilitators for medication adherence. Clinical Medicine \& Research, 9(3-4), 157. doi:10.3121/ cmr.2011.1020.ps2-32

Rosenstock, I. M., Strecher, V. J., \& Becker, M. H. (1988). Social learning theory and the health belief model. Health Education Quarterly, 15(2), 175-183. doi:10.1177/109019818801500203 PMID:3378902

Ruddy, K., Mayer, E., \& Partridge, A. (2009). Patient adherence and persistence with oral anticancer treatment. CA: a Cancer Journal for Clinicians, 59(1), 56-66. doi:10.3322/caac.20004 PMID:19147869

Safren, S. A., Hendriksen, E. S., Desousa, N., Boswell, S. L., \& Mayer, K. H. (2003). Use of an on-line pager system to increase adherence to antiretroviral medications. AIDS Care, 15(6), 787-793. doi:10.1080/0954012 0310001618630 PMID:14617500 
Samet, J. H., Horton, N. J., Meli, S., Freedberg, K. A., \& Palepu, A. (2004). Alcohol consumption and antiretroviral adherence among HIV-infected persons with alcohol problems. Alcoholism, Clinical and Experimental Research, 28(4), 572-577. doi:10.1097/01.ALC.0000122103.74491.78 PMID:15100608

Santo, K., Richtering, S. S., Chalmers, J., Thiagalingam, A., Chow, C. K., \& Redfern, J. (2016). Mobile Phone Apps to Improve Medication Adherence: A Systematic Stepwise Process to Identify High-Quality Apps. JMIR mHealth and uHealth, 4(4), e132. doi:10.2196/mhealth.6742 PMID:27913373

Sayegh, C. S., Szmuszkovicz, J. R., Menteer, J., Sherer, S., Thomas, D., Lestz, R., \& Belzer, M. (2018). Cell phone support to improve medication adherence among solid organ transplant recipients. Pediatric Transplantation, 22(6), e13235. doi:10.1111/petr.13235 PMID:29920879

Schectman, J. M., Bovbjerg, V. E., \& Voss, J. D. (2002). Predictors of medication-refill adherence in an indigent rural population. Medical Care, 4O(12), 1294-1300. doi:10.1097/00005650-200212000-00016 PMID:12458310

Shet, A., Arumugam, K., Rodrigues, R., Rajagopalan, N., Shubha, K., Raj, T., D'souza, G., \& De Costa, A. (2010). Designing a mobile phone-based intervention to promote adherence to antiretroviral therapy in South India. AIDS and Behavior, 14(3), 716-720. doi:10.1007/s10461-009-9658-3 PMID:20054634

Shiyanbola, O. O., Unni, E., Huang, Y. M., \& Lanier, C. (2018). The association of health literacy with illness perceptions, medication beliefs, and medication adherence among individuals with type 2 diabetes. Research in Social \& Administrative Pharmacy, 14(9), 824-830. doi:10.1016/j.sapharm.2017.12.005 PMID:29317189

Siegel, D., Lopez, J., \& Meier, J. (2007). Antihypertensive medication adherence in the Department of Veterans Affairs. The American Journal of Medicine, 120(1), 26-32. doi:10.1016/j.amjmed.2006.06.028 PMID:17208076

Silavanich, V., Nathisuwan, S., Phrommintikul, A., \& Permsuwan, U. (2019). Relationship of medication adherence and quality of life among heart failure patients. Heart \& Lung, 48(2), 105-110. doi:10.1016/j. hrtlng.2018.09.009 PMID:30384984

Simoni, J. M., Pearson, C. R., Pantalone, D. W., Marks, G., \& Crepaz, N. (2006). Efficacy of interventions in improving highly active antiretroviral therapy adherence and HIV-1 RNA viral load: a meta-analytic review of randomized controlled trials. Journal of Acquired Immune Deficiency Syndromes, 43(1), S23.

Sirey, J. A., Bruce, M. L., Alexopoulos, G. S., Perlick, D. A., Friedman, S. J., \& Meyers, B. S. (2001). Stigma as a barrier to recovery: Perceived stigma and patient-rated severity of illness as predictors of antidepressant drug adherence. Psychiatric Services (Washington, D.C.), 52(12), 1615-1620. doi:10.1176/appi.ps.52.12.1615 PMID:11726752

Smith, A., Krishnan, J. A., Bilderback, A., Riekert, K. A., Rand, C. S., \& Bartlett, S. J. (2006). Depressive symptoms and adherence to asthma therapy after hospital discharge. Chest, 130(4), 1034-1038. doi:10.1378/ chest.130.4.1034 PMID:17035435

Sokol, M. C., McGuigan, K. A., Verbrugge, R. R., \& Epstein, R. S. (2005). Impact of medication adherence on hospitalization risk and healthcare cost. Medical Care, 43(6), 521-530. doi:10.1097/01.mlr.0000163641.86870. af PMID:15908846

Spaan, P., van Luenen, S., Garnefski, N., \& Kraaij, V. (2018). Psychosocial interventions enhance HIV medication adherence: A systematic review and meta-analysis. Journal of Health Psychology. Advance online publication. doi:10.1177/1359105318755545 PMID:29417851

Spilker, B., \& Cramer, J. A. (1991). Patient compliance in medical practice and clinical trials. Academic Press.

Sposaro, F., \& Tyson, G. (2009). iFall: an Android application for fall monitoring and response. 2009 Annual International Conference of the IEEE Engineering in Medicine and Biology Society, 6119-6122. doi:10.1109/ IEMBS.2009.5334912

Stirratt, M. J., Curtis, J. R., Danila, M. I., Hansen, R., Miller, M. J., \& Gakumo, C. A. (2018). Advancing the Science and Practice of Medication Adherence. Journal of General Internal Medicine, 33(2), 216-222. doi:10.1007/s11606-017-4198-4 PMID:29204969

Stoehr, G. P., Lu, S.-Y., Lavery, L., Vander Bilt, J., Saxton, J. A., Chang, C.-C. H., \& Ganguli, M. (2008). Factors associated with adherence to medication regimens in older primary care patients: The Steel Valley Seniors Survey. The American Journal of Geriatric Pharmacotherapy, 6(5), 255-263. doi:10.1016/j.amjopharm.2008.11.001 PMID:19161928 
Sullivan, S. D. (1990). Noncompliance with medication regimens and subsequent hospitalization: A literature analysis and cost of hospitalization estimate. J Res Pharm Econ, 2, 19-33.

Sung, J. C., Nichol, M. B., Venturini, F., Bailey, K. L., McCombs, J. S., \& Cody, M. (1998). Factors affecting patient compliance with antihyperlipidemic medications in an HMO population. The American Journal of Managed Care, 4(10), 1421-1430. PMID:10338735

Thakkar, J., Kurup, R., Laba, T. L., Santo, K., Thiagalingam, A., Rodgers, A., Woodward, M., Redfern, J., \& Chow, C. K. (2016). Mobile telephone text messaging for medication adherence in chronic disease a metaanalysis. JAMA Internal Medicine, 176(3), 340-349. doi:10.1001/jamainternmed.2015.7667 PMID:26831740

Thorpe, C. T., Bryson, C. L., Maciejewski, M. L., \& Bosworth, H. B. (2009). Medication acquisition and self-reported adherence in veterans with hypertension. Medical Care, 47(4), 474-481. doi:10.1097/ MLR.0b013e31818e7d4d PMID:19330891

Trevino, J., Albright, T., Wright, F., \& Cigarroa, L. (2005). Correlates of medication knowledge and adherence: Findings from the residency research network of South Texas. Family Medicine, 37(10), 712-718. PMID:16273450

Trivedi, R. B., Ayotte, B., Edelman, D., \& Bosworth, H. B. (2008). The association of emotional well-being and marital status with treatment adherence among patients with hypertension. Journal of Behavioral Medicine, 31(6), 489-497. doi:10.1007/s10865-008-9173-4 PMID:18780175

Turner, A., Hochschild, A., Burnett, J., Zulfiqar, A., \& Dyer, C. B. (2012). High prevalence of medication nonadherence in a sample of community-dwelling older adults with adult protective services-validated self-neglect. Drugs \& Aging, 29(9), 741-749. doi:10.1007/s40266-012-0007-2 PMID:23018610

Urquhart, J. (1997). The electronic medication event monitor. Clinical Pharmacokinetics, 32(5), $345-356$. doi:10.2165/00003088-199732050-00001 PMID:9160169

Vawter, L., Tong, X., Gemilyan, M., \& Yoon, P. W. (2008). Barriers to antihypertensive medication adherence among adults-United States, 2005. Journal of Clinical Hypertension, 10(12), 922-929. doi:10.1111/j.17517176.2008.00049.x PMID:19120718

Vervloet, M., Linn, A. J., van Weert, J. C. M., De Bakker, D. H., Bouvy, M. L., \& Van Dijk, L. (2012). The effectiveness of interventions using electronic reminders to improve adherence to chronic medication: A systematic review of the literature. Journal of the American Medical Informatics Association: JAMIA, 19(5), 696-704. doi:10.1136/amiajnl-2011-000748 PMID:22534082

Vervloet, M., van Dijk, L., Santen-Reestman, J., Van Vlijmen, B., Van Wingerden, P., Bouvy, M. L., \& de Bakker, D. H. (2012). SMS reminders improve adherence to oral medication in type 2 diabetes patients who are real time electronically monitored. International Journal of Medical Informatics, 81(9), 594-604. doi:10.1016/j. ijmedinf.2012.05.005 PMID:22652012

Vik, S. A., Maxwell, C. J., \& Hogan, D. B. (2004). Measurement, correlates, and health outcomes of medication adherence among seniors. The Annals of Pharmacotherapy, 38(2), 303-312. doi:10.1345/aph.1D252 PMID: 14742770

Vlasnik, J. J., Aliotta, S. L., \& DeLor, B. (2005). Medication adherence: Factors influencing compliance with prescribed medication plans. The Case Manager, 16(2), 47-51. doi:10.1016/j.casemgr.2005.01.009 PMID:15818344

Voils, C. I., Steffens, D. C., Bosworth, H. B., \& Flint, E. P. (2005). Social support and locus of control as predictors of adherence to antidepressant medication in an elderly population. The American Journal of Geriatric Psychiatry, 13(2), 157-165. doi:10.1097/00019442-200502000-00010 PMID:15703325

Vrijens, B. (2019). A Six Sigma framework to successfully manage medication adherence. British Journal of Clinical Pharmacology, 85(8), 1661-1663. doi:10.1111/bcp.13905 PMID:30834553

Vrijens, B., Antoniou, S., Burnier, M., de la Sierra, A., \& Volpe, M. (2017). Current situation of medication adherence in hypertension. Frontiers in Pharmacology, 8. doi:10.3389/fphar.2017.00100 PMID:28298894

Wang, P. S., Bohn, R. L., Knight, E., Glynn, R. J., Mogun, H., \& Avorn, J. (2002). Noncompliance with antihypertensive medications. Journal of General Internal Medicine, 17(7), 504-511. doi:10.1046/j.15251497.2002.00406.x PMID:12133140 
Waterhouse, D. M., Calzone, K. A., Mele, C., \& Brenner, D. E. (1993). Adherence to oral tamoxifen: A comparison of patient self-report, pill counts, and microelectronic monitoring. Journal of Clinical Oncology, 11(6), 1189-1197. doi:10.1200/JCO.1993.11.6.1189 PMID:8501505

Weingart, S. N., Brown, E., Bach, P. B., Eng, K., Johnson, S. A., Kuzel, T. M., Langbaum, T. S., Leedy, R. D., Muller, R. J., Newcomer, L. N., O'Brien, S., Reinke, D., Rubino, M., Saltz, L., \& Walters, R. S. (2008). NCCN Task Force Report: Oral chemotherapy. Journal of the National Comprehensive Cancer Network: JNCCN, 6(S3), S1-S14. doi:10.6004/jnccn.2008.2003 PMID:18377852

Wells, K., Pladevall, M., Peterson, E. L., Campbell, J., Wang, M., Lanfear, D. E., \& Williams, L. K. (2008). Race-ethnic differences in factors associated with inhaled steroid adherence among adults with asthma. American Journal of Respiratory and Critical Care Medicine, 178(12), 1194-1201. doi:10.1164/rccm.200808-1233OC PMID:18849496

Williams, A., Manias, E., \& Walker, R. (2008). Interventions to improve medication adherence in people with multiple chronic conditions: A systematic review. Journal of Advanced Nursing, 63(2), 132-143. doi:10.1111/ j.1365-2648.2008.04656.x PMID:18537843

Williams, G. C., Rodin, G. C., Ryan, R. M., Grolnick, W. S., \& Deci, E. L. (1998). Autonomous regulation and long-term medication adherence in adult outpatients. Health Psychology, 17(3), 269-276. doi:10.1037/02786133.17.3.269 PMID:9619477

Wohlers, E. M., Sirard, J. R., Barden, C. M., \& Moon, J. K. (2009). Smart phones are useful for food intake and physical activity surveys. 2009 Annual International Conference of the IEEE Engineering in Medicine and Biology Society, 5183-5186. doi:10.1109/IEMBS.2009.5333721

Wroe, A. L. (2002). Intentional and unintentional nonadherence: A study of decision making. Journal of Behavioral Medicine, 25(4), 355-372. doi:10.1023/A:1015866415552 PMID:12136497

Wroth, T. H., \& Pathman, D. E. (2006). Primary medication adherence in a rural population: The role of the patient-physician relationship and satisfaction with care. Journal of the American Board of Family Medicine, 19(5), 478-486. doi:10.3122/jabfm.19.5.478 PMID:16951297

Wu, J.-R., Chung, M., Lennie, T. A., Hall, L. A., \& Moser, D. K. (2008). Testing the psychometric properties of the Medication Adherence Scale in patients with heart failure. Heart \& Lung: The Journal of Acute and Critical Care, 37(5), 334-343. doi:10.1016/j.hrtlng.2007.10.001 PMID:18790334

Wu, R., Rossos, P., Quan, S., Reeves, S., Lo, V., Wong, B., Cheung, M., \& Morra, D. (2011). An evaluation of the use of smartphones to communicate between clinicians: A mixed-methods study. Journal of Medical Internet Research, 13(3), e59. doi:10.2196/jmir.1655 PMID:21875849

Xie, Z., Clair, P. S., Goldman, D. P., \& Joyce, G. (2019). Racial and ethnic disparities in medication adherence among privately insured patients in the United States. PLoS One, 14(2). Advance online publication. doi:10.1371/ journal.pone.0212117 PMID:30763400

Yap, A. F., Thirumoorthy, T., \& Kwan, Y. H. (2016). Medication adherence in the elderly. Journal of Clinical Gerontology and Geriatrics, 7(2), 64-67. doi:10.1016/j.jcgg.2015.05.001

Zed, P. J., Abu-Laban, R. B., Balen, R. M., Loewen, P. S., Hohl, C. M., Brubacher, J. R., Wilbur, K., Wiens, M. O., Samoy, L. J., Lacaria, K., \& Purssell, R. A. (2008). Incidence, severity and preventability of medicationrelated visits to the emergency department: A prospective study. Canadian Medical Association Journal, 178(12), 1563-1569. doi:10.1503/cmaj.071594 PMID:18519904

Zedler, B. K., Kakad, P., Colilla, S., Murrelle, L., \& Shah, N. R. (2011). Does packaging with a calendar feature improve adherence to self-administered medication for long-term use? A systematic review. Clinical Therapeutics, 33(1), 62-73. doi:10.1016/j.clinthera.2011.02.003 PMID:21397775 
Saibal Kumar Saha (UGC NET Qualified) holds a first class Master's degree in MBA and a first class Bachelor's Degree in Electronics and Communication Engineering. He has 8+ years of experience and has worked in MNCs like Cognizant Technology Solutions and Tata Aig Life. He has served National Institute of Technology, Silchar; Jyotirmoy School of Business, Kolkata; University of Technology and Management, Shillong; and is presently working as Assistant Professor at Sikkim Manipal Institute of Technology, Majitar, India.

Anindita Adhikary $(P h D)$ is currently a Professor in Management, Sikkim Manipal University, India. A commerce graduate from Gauhati University, India, she happens to be an MBA from Tezpur Central University, India and was awarded Doctorate by Gauhati University in 2009. Dr. Adhikary has 20 years of professional experience in academics and corporate sector. She has 65+ research publications (select papers in Scopus) to her credit and has been abroad a number of times in order to have an enriched exposure at international level. Dr. Adhikary had participated in 25 professional workshops and delivered talks as Guest Speaker at different Orientation Programmes initiated through National Productivity Council, MSME, Govt. of India and Department of Commerce. Govt. of Sikkim. Her domain of interest includes Finance and International Trade.

Ajeya Jha is a professor at the Department of Management Studies at Sikkim Manipal Institute of Technology (SMIT) since February 2000. In addition to having post-graduate and doctoral degrees in Business Administration, he possesses graduation and post graduation in Pharmaceutical Sciences also. fellow of International Society of Management. Has a rich and diverse industrial experience of 15 years (includes launching two green-field projects), teaching 22 years and with NGO 8 months. He is the founder Chief coordinator of Entrepreneurship Development Cell (EDC) at SMIT established with support from Department of Science and Technology, Government of India. He has 20 books, 150 research papers and 15 book chapters to his credit.

Vijay Kumar Mehta, PhD, MBBS, MD, PGDCA, is currently working as the Dean of Sikkim Manipal Institute of Medical Sciences. After retiring from Indian Armed Forces as Brigadier on 30th Sept. 2013 joined Sikkim Manipal Institute of Medical Sciences, Gangtok on 21st Oct. 2013 as Professor in the department of Community Medicine. Subsequently, took over as Professor and Head department of Community Medicine at Sikkim Manipal Institute of Medical Sciences, Gangtok on 1st Feb. 2014. Have more than 22 years of teaching experiences including twelve and half years as Professor and 5 years as Professor and HOD Community Medicine in SMIMS, Gangtok. 